\title{
Free-electron laser inverse-Compton interaction $x$-ray source
}

\author{
Pardis Niknejadi, ${ }^{1, *}$ Jeremy M. D. Kowalczyk, ${ }^{1, \dagger}$ Michael R. Hadmack, ${ }^{1,2}$ \\ Bryce T. Jacobson, ${ }^{1,3}$ Ian Howe, ${ }^{1}$ Shidong Kan, ${ }^{1}$ Steven Smith, ${ }^{1}$ Eric B. Szarmes, ${ }^{1}$ \\ Gary Varner, ${ }^{1}$ and John M. J. Madey, \\ ${ }^{1}$ University of Hawai 'i at Mānoa, Department of Physics and Astronomy, Honolulu, Hawaii 96822, USA \\ ${ }^{2}$ Oceanit, 828 Fort Street Mall, Honolulu, Hawaii 96813, USA \\ ${ }^{3}$ SLAC National Accelerator Laboratory, 2575 Sand Hill Road, Menlo Park, California 94025, USA
}

(Received 11 February 2019; published 29 April 2019)

\begin{abstract}
Free-electron lasers (FEL) and synchrotron sources of high brilliance x-rays have proven to be of tremendous value in basic and applied research. Inverse-Compton sources (ICS) can achieve brilliance matching the requirements of many applications pioneered at those FEL and synchrotron facilitiesincluding phase contrast imaging, macromolecular x-ray crystallography, and x-ray microscopy-but with size, cost, and complexity compatible with a small laboratory. The free-electron laser inverseCompton interaction compact x-ray source at the University of Hawaii at Manoa is a unique approach to an ICS which employs an FEL as the laser source. We have measured a total average flux of $3.0 \times 10^{5}$ photons/second with an average brilliance of $2.0 \times 10^{7}$ photons $/ \mathrm{s} \mathrm{mm}^{2} \mathrm{mrad}^{2} 0.1 \%$ of bandwidth (BW) with a peak energy of $10.9 \mathrm{keV}$ from the source. While these results are modest in comparison to the standards set by other IC sources, upgrades to the system have the potential to increase the total average flux to $9.2 \times 10^{11}$ photons/second with an average brilliance of $1.9 \times$ $10^{12}$ photons $/ \mathrm{s} \mathrm{mm}^{2} \mathrm{mrad}^{2} 0.1 \% \mathrm{BW}$ : comparing more favorably to other sources. We discuss the scientific program, the progress made in design and development, and the achievements of the source to date. We also outline future upgrades and integration needed to yield an enabling source for emerging high brilliance x-ray applications.
\end{abstract}

DOI: 10.1103/PhysRevAccelBeams.22.040704

\section{INTRODUCTION}

\section{A. Motivation}

The Linac Coherent Light Source (LCLS) at SLAC National Accelerator Laboratory and other advanced x-ray FEL and synchrotron light sources have proven the value of high brilliance $\mathrm{x}$-rays for basic and applied research [1], but these sources cannot be used on a daily basis by a particular user. Inverse-Compton ${ }^{1} \mathrm{x}$-ray sources (ICS) are consistent in size, cost, and operational complexity with small

\footnotetext{
*Present address: Deutsche Elektronen-Synchrotron (DESY), Notkestraße 85, 22607 Hamburg, Germany.

pardis@hawaii.edu

jeremymk@hawaii.edu

Deceased.

${ }^{1}$ Inverse-Compton scattering, relativistic Thomson scattering, Compton backscattering, and laser Compton scattering are all commonly used in literature and are equivalent in our discussion of $\mathrm{x}$ - and gamma-ray sources.
}

Published by the American Physical Society under the terms of the Creative Commons Attribution 4.0 International license. Further distribution of this work must maintain attribution to the author(s) and the published article's title, journal citation, and DOI. laboratories (e.g., universities, hospitals, or semiconductor manufacturing facilities) making the benefits of high brilliance x-rays-previously available only at the national laboratory scale facilities-available for daily use to a broader range of users [2]. These sources have applications emerging in many sectors including nuclear materials detection [3], small-angle x-ray scattering [4], phase contrast imaging [5], macromolecular x-ray crystallography for drug discovery [6], and x-ray microscopy [7].

In these ICS systems, high intensity laser pulses collide with electron bunches to produce $\mathrm{x}$-rays with flux proportional to the product of the laser peak power and the average current of the electron beam (ebeam). Maximizing the laser peak power, through the use of a modest power GHz laser and optical storage cavity, allows for production of useful $\mathrm{x}$-ray flux with a lower current electron beam. The storage cavity round-trip time for the circulating pulses at $\mathrm{GHz}$ rates requires cavity lengths of $\sim 10 \mathrm{~cm}$. To maximize the $\mathrm{x}$-ray flux produced, the electron beam rep rate should match the cavity circulation rate, enabling collision of the laser and electron pulses on each pass through the cavity. So $\mathrm{GHz}$ lasers and ebeams allow the use of inexpensive short cavities with small mirrors. Enhancing the laser peak power by stacking pulses in a cavity permits the use of a low peak 
power pump laser while achieving the high circulating peak optical powers needed to produce useful x-ray flux. Essentially, as detailed in our earlier SPIE paper [8], in the cavity-enhanced inverse-Compton approach, the $\mathrm{x}$-ray flux is maximized while the cost is minimized.

The combination of a free-electron laser (FEL) and a thermionic microwave electron gun appears to be an optimal solution for a cavity-enhanced x-ray source. While substantial progress has been recently made on $\mathrm{GHz}$ rep-rate solid state laser systems, employing an FEL to create a GHz pump laser using the same ebeam needed for the inverse-Compton interaction eliminates the need for such a solid state laser system. The thermionic gun is a mature technology with high reliability, low cost, and the capability to provide electron bunches at $\mathrm{GHz}$ rep rate with the high average and the peak current needed for an optimal cavity-enhanced ICS. An FEL pump driven by the same electron beam used for the inverse-Compton interaction has the added benefit of intrinsic synchronization between the laser and $\mathrm{GHz}$ rep-rate electron beam. Most current ICS approaches are restricted to sub-GHz repetition rates [9-11], and electron guns capable of $\mathrm{GHz}$ rep rates (for example [12]) have yet to be employed in ICS systems.

\section{B. Scientific goal}

The purpose of free-electron laser inverse-Compton interaction (FELICIA) is to explore the physics and technology most critical to the development of optical storage cavity-based ICS sources with the eventual goal of evaluating a differential absorption spectroscopy technique at $\mathrm{x}$-ray and gamma-ray wavelengths. The initial exploration for this purpose can be divided into three categories.

(1) Generation of the electron and laser beams with appropriate parameters and configuration of the Compton interaction point (IP). This was broken into two parts: (a) design and development of the electron beam focusing system and the necessary diagnostic and control elements required to focus the electron beam to a spot size smaller than $100 \mu \mathrm{m}$ at the plane in which the electrons collide with the counterpropagating optical pulses from the systems laser light source; (b) optimization of the IR FEL to produce a high repetition rate, phase-locked laser beam suitable for pumping a storage cavity.

(2) Design and development of the storage cavity and experimental demonstration of the increased intensities of tunable $\mathrm{x}$-rays and gamma rays made possible through the use of an optical storage cavity to provide the high level of circulating optical power required for efficient $\mathrm{x}$ - and gamma-ray generation via inverse Compton scattering. Fundamental differences between our approach and other projects such as the Duke HIGS Laboratory [13] are discussed in Sec. IV B.
(3) The development and demonstration of the fast ( picosecond) detector and data acquisition systems needed for time-resolved characterization of the ICS $\mathrm{x}$-ray and gamma ray output.

The implementation of these three categories was divided into three phases: phase 0 -initial designs and beam line upgrade; phase 1-development of (1), while working on design and bench test of (2) and (3), and demonstrating a system that is capable of achieving average $\mathrm{x}$-ray flux on the order of $10^{6}$ photons per second at the IP in a singlepass setup without the optical storage cavity; phase 2completion and integration of (2) and (3), to achieve production rates above $10^{11}$ photons per second.

The objective of this paper is to give an account of what has been accomplished so far (phases 0 and 1). In Sec. II, we will describe the University of Hawaii FEL beam line and technical design and modification needed for FELICIA. In Sec. III, the setup for the single-pass experiment and initial results are included. Section IV gives an account of scientific and technological milestones made towards the proposed high-flux setup and addresses methods of overcoming some of the inherent limitations of the system. Developments associated with (3), the design and test of a ps X-ray diagnostics and readout system, will be the subject of future publications.

\section{UNIVERSITY OF HAWAII FEL BEAM LINE AND TECHNICAL DESIGN FOR FELICIA}

The rf linac, undulator, and resonator optics (originally the MkIII free electron laser system at Stanford) were transferred to UH in 2006 and recommissioned as part of the Mark V (MkV) FEL ${ }^{2}$ laboratory which includes newly upgraded capabilities. The MkV beam line includes a tenmeter-long chicane for diagnostics and phase space tomography while also allowing ample room for insertion devices like an ICS. The MkV satisfies many of the critical requirements to demonstrate the feasibility of an infrared-FEL-based compact $\mathrm{x}$ - or gamma-ray source with high average power. The present configuration (see Fig. 1), with a $\sim 40 \mathrm{MeV}$ electron beam, converts the infrared output of the FEL to the $\mathrm{x}$-ray regime at photon energies $\sim 10 \mathrm{keV}$ (FELICIA).

Both the electron and laser beam must meet stringent criteria for the inverse-Compton scattering process to produce the desired flux and source brightness. We discuss the requirements on beam current, energy spread, stability of laser amplitude within a pulse, alignment, and matching of the electron beam, and the way these requirements were achieved in this section.

\footnotetext{
${ }^{2}$ The Mark IV (MkIV) FEL was an IR FEL at Stanford University similar to the MkIII but in a MOPA configuration, hence the gap in numbering.
} 


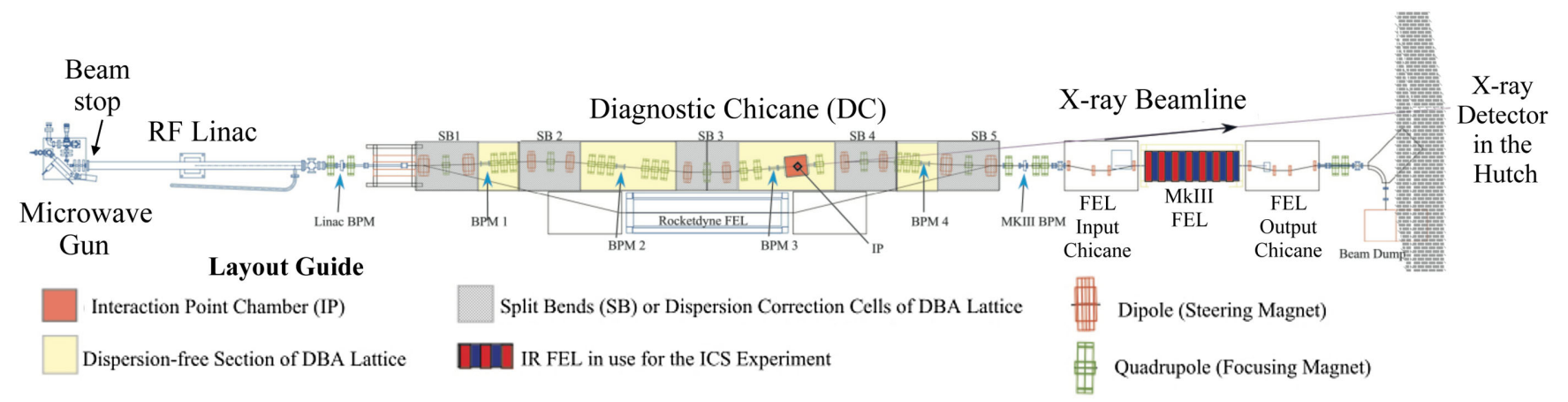

FIG. 1. Annotated scale drawing of the MkV FEL beam line in FELICIA configuration where each split bend contains an OTR screen for diagnotics.

\section{A. Microwave gun}

FELICIA's electron gun is normally conducting and utilizes a $\mathrm{LaB}_{6}$ thermionic cathode and Enge alpha magnet [14] for bunch compression and momentum selection (via a knife edge) (see Fig. 2). The details of the gun have been described previously [15-17]. A tungsten heater filament on the back of the cathode keeps it at a nominal operating temperature of $\sim 1700 \mathrm{~K}$. The system is designed to deliver $\sim 170 \mathrm{~mA}$ of average current to the linac during a $4.5 \mu \mathrm{s}$

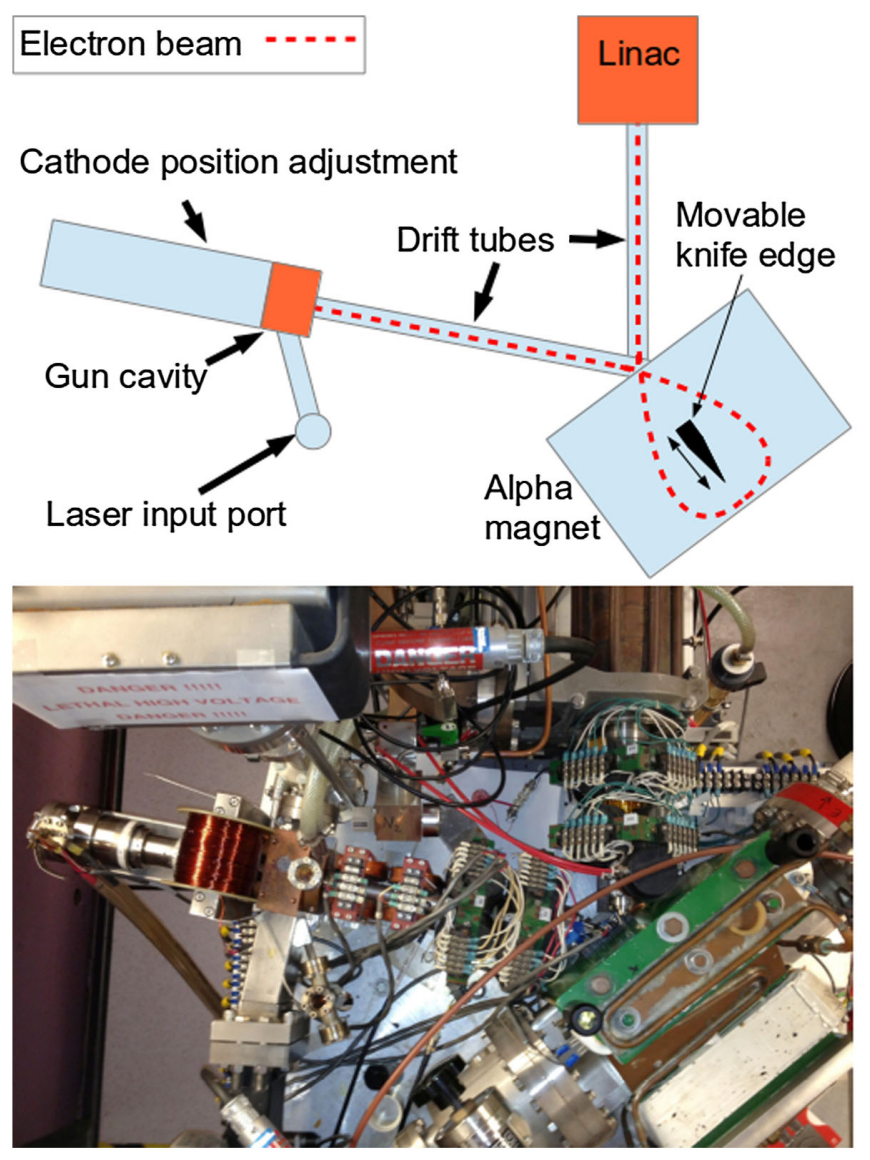

FIG. 2. Diagram (top) and image (bottom) of the microwave thermionic electron gun and alpha magnet. macropulse at $100 \mathrm{~Hz}$ (bunch charge of $60 \mathrm{pC}$, time average current of $72 \mu \mathrm{A}$ ), with bunches of approximately 1-2 ps rms [18]. Typically we operate the system at $4 \mathrm{~Hz}$ for testing (see Fig. 3 for timing structure of the pulse in this mode). We studied the bunch compression and limitations of the macropulse length due to back-bombardment heating previously [19]. The gun delivers electron bunches at $2856 \mathrm{MHz}$ repetition rates during the macropulse, but due to the maximum macropulse repetition rate of $100 \mathrm{~Hz}$, the average repetition rate is only $1.43 \mathrm{MHz}$. Due to its high average current, this gun is still competitive in comparison with modern photocathode guns [20]. Additionally, the thermionic gun is less complex, has far longer cathode lifetimes, and is available at much lower cost. Nonetheless, to truly take advantage of the capabilities of cavityenhanced ICS sources, the limitation on the length of the $\mathrm{GHz}$ train of electron pulses (the macropulse) inherent in microwave guns must be addressed. We discuss in Sec. IVA how a fourfold increase in the average current may be possible by employing a laser cathode-cooling system.

The nominal temperature of the cathode for operation is 1700 Kelvin. However, the cathode surface will gradually develop an oxide layer at this temperature. Increasing the temperature to $1750 \mathrm{~K}$ can remove the oxide layer [21] so the cathode is baked out at $1750 \mathrm{~K}$ before the start of

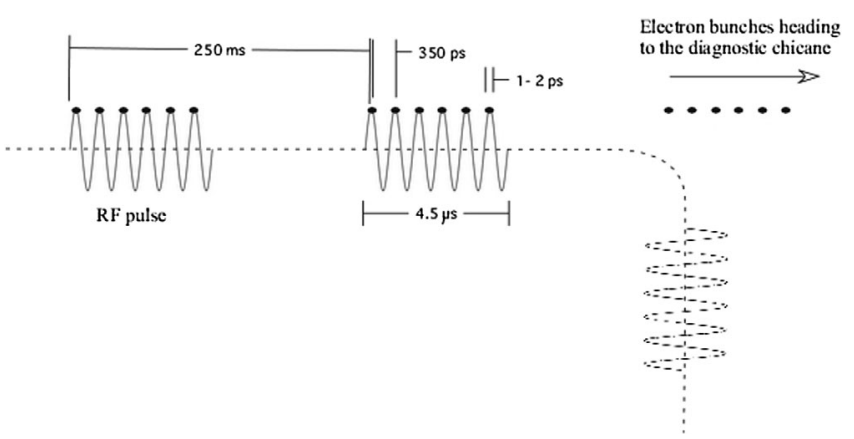

FIG. 3. Timing structure of the electron micro- and macropulses. 


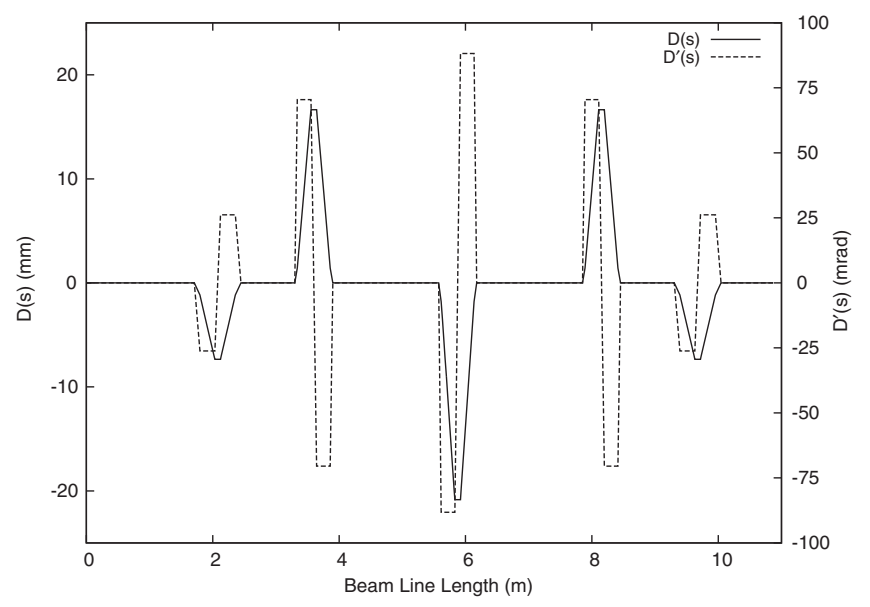

FIG. 4. Diagnostic chicane dispersion functions.

operations each day. We redesigned the tungsten filament in the cathode heater with extra loops for higher resistance, allowing the cathode to reach these elevated temperatures with lower current.

\section{B. Matching electron beam parameters 1. Diagnostic chicane (DC)}

The design for DC was inspired by the double-bend achromat (DBA), first proposed by Panofsky and then by Chasman and Green [22] for the bright circulating beam of the National Synchrotron Light Source at Brookhaven National Laboratory (BNL). The Chasman-Green DBA is used in the lattice of many modern synchrotron and storage rings. In a circular machine, the bend angles need to add up to $360^{\circ}$. By alternating the direction of the bends in the Chasman-Green DBA lattice design, the ring-lattice unfolds, making it a suitable design lattice for a linear accelerator and transport line. In the MkV DC, the bend angles and drift lengths of the DBA sections are determined by many factors, including the orientation angle of the focusing sections, the availability of space in the lab [23], the constrained location of the x-ray extraction beam line, and more which makes the design a complicated task. The MkV DC lattice was designed and fully commissioned in 2012 [24]. This design consists of a series of five pairs of dipole magnets with integrated vacuum chambers and a single dispersion-correcting quadrupole midway between the dipole pair. The resulting beam line is comprised of five alternating so-called "split bends" (SB1-SB5). These bends are spaced approximately equally along the section of the transport system between the linac and the FEL. In Fig. 1, the dispersion-correcting sections (split bends) of the lattice have been identified with the dispersion-free focusing sections in between. Figures 4 and 5 show the dispersion functions and the beam envelopes from the DC to the undulator for the designed configuration.

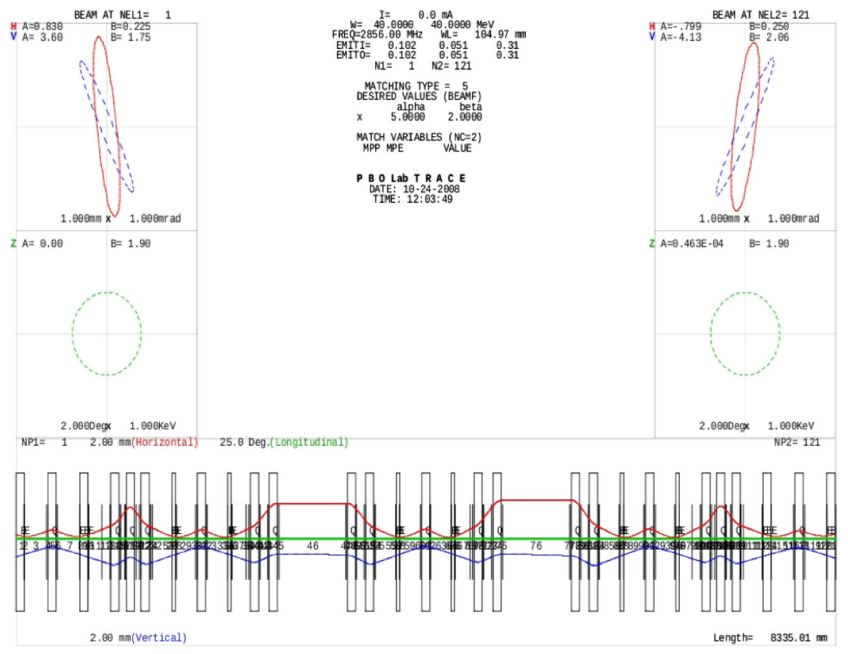

FIG. 5. Horizontal and vertical beam envelopes for the designed configuration with the PBOLab software.

\section{Beam requirement}

For FELICIA, where the electron beam also pumps the IR FEL, in addition to the current, emittance and energy spread requirements, the beam profile must match the requirements of the interaction region and the IR FEL simultaneously. The probability of a single photon scattered by ICS increases as the electron beam radius $\left(w_{0}\right)$ gets smaller. Therefore it is essential to focus the beam to a waist at the IP. At the same time, since the same beam will be driving the FEL, over focusing the beam at the IP must be avoided to ensure nonlinear and undesirable effects are not introduced. The operation of the FEL depends on the ability of the electron beam to deposit energy in the laser field. Therefore, the electron beam envelope must match the optical mode of the FEL both spatially and temporally. Consequently, maintaining a well-focused beam is critical. One advantage of the $\mathrm{MkV}$ diagnostic chicane is that it can accommodate the isochronous temporal match between ebeam and laser by canceling the dispersion caused by offenergy electrons in the split bends. For the spatial match, we have the following two requirements: (1) the optical mode and the electron beam must be aligned horizontally and vertically (in other words, share a common axis) and (2) they must also have the same phase-space configuration (transverse size and angular spread) at the interaction point.

For a vertical match to the undulator, we want the vertical section of the electron beam to propagate as a collimated pencil beam, the electron beam must enter the undulator at a waist $\beta_{y} \equiv \frac{w_{y}}{\theta_{y} m}=\frac{\gamma}{\hat{K} k_{u}}$, where $\hat{K}, k_{u}$, and $\gamma$ are respectively the undulator parameter, undulator wave number, and the relativistic mass factor. To maximize gain, we should keep the horizontal radius of the electron beam to a minimum (smaller than the optical radius $\epsilon_{x} \ll \lambda[25]$ ). The first relation can be evaluated for the MkV FEL, using $\hat{K}=1.2$ and $\lambda_{u}=2.3 \mathrm{~cm}$ and assuming a normalized emittance of 
$8 \pi \mathrm{mm}$ mrad, we find that we would need a $y_{\max } \approx 110 \mu \mathrm{m}$ which will have a $y_{\max }^{\prime} \approx 0.03 \mathrm{mrad}$. The horizontal requirement is easily met with the parameters of the MkV FEL. We assume that the beam is drifting with a Gaussian profile. Using $\frac{\epsilon_{x}}{\gamma}=x_{\max } \cdot \theta$, where $\theta=\frac{x_{\max }}{z_{R}}$ and $z_{R}$ is the Rayleigh length and equal to half the undulator length. We can expect a horizontal beam spot of $140 \mu \mathrm{m}$. We utilized a screen located before the first chicane magnets $(\pi / 2$ screen) for measuring and tuning the match into the undulator. This screen is positioned so that a vertical waist and a minimum horizontal beam on this screen would automatically input a vertical waist and minimum horizontal beam [26].

\section{Diagnostic and control elements}

Both microfocus and undulator matching requirements of the electron beam demand a well-centered beam. For the purpose of alignment, there are multiple beam profile monitors comprised of optical transition radiation (OTR) screens and beam position monitors (BPMs) installed along the DC. Figure 1 shows the placement of these elements. The placement of these BPMs is important for their optimal performance. Two varieties of BPMs are installed along the $\mathrm{MkV}$ beam line, a wall current type and a strip-line type. The wall current BPMs carry induced wall current at four cardinal positions around the beam pipe with a minimum of temporal distortion to a four-port connector block. There are two wall current BPMs on the beam line: one is placed between the linac quads (before the DC) and the other is placed before the MkIII undulator (after the DC). The DC includes a series of periodically spaced (SLAC-type) stripline BPMs. The strip-line BPMs consist of a stainless steel body with four copper electrodes oriented with $\pi / 2$ symmetry [27].

Although the DC was designed in-house [24] and its matrix is known, due to the lack of periodicity, finding a real analytical solution that describes the beam line is not possible. Prepackaged simulation codes are often used to describe such beam lines, but in our case, due to the high number of elements and constraints, the parameter search space is large. If the system is not being operated close to a configuration set by the simulation, it is difficult to find a stable solution. For this reason, one of the most important diagnostic and control elements for the $\mathrm{MkV}$ is a computerbased beam transport simulator module (TSM). The TSM, an on-line beam modeling system, gets its inputs from the power supplies that provide the currents to the quadruples installed along the diagnostic chicane and, given a set of initial beam parameters, it can estimate the electron beam envelope's evolution through the DC [26]. The initial beam parameters are determined using the beam profiles measured with the OTR monitors for different cathode positions which offer collimated, diverging and converging beam to the DC, and analyzed with the TSM. These input values were consistently more accurate than the ones achieved by

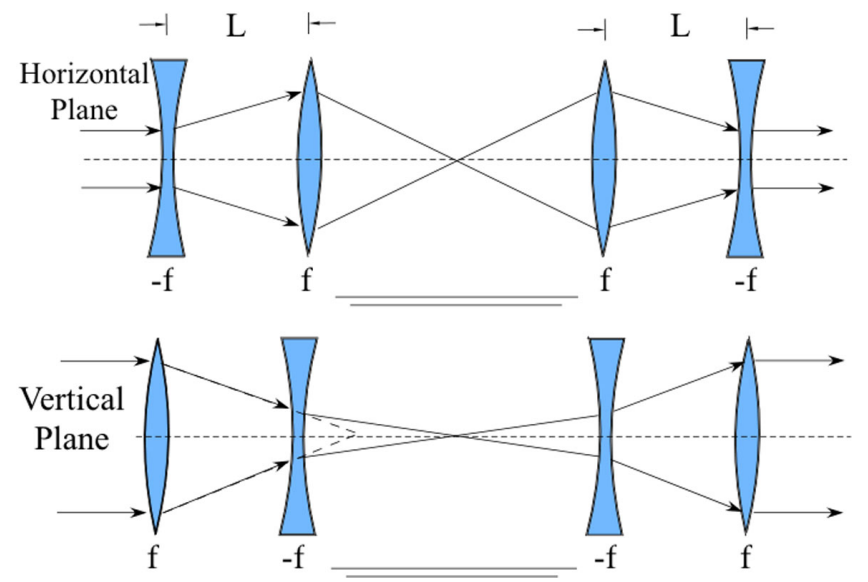

FIG. 6. Demonstration of the electron beam focusing requirement at the IP using ray optics. The double quads before and after the IP in each plane are represented by a pair of converging and diverging lenses.

traditional quad scan measurements. It was also revealed that the system's electron gun could best serve the functions of the transport system, IP focusing quads, and the IR FEL when the gun's cathode was moved to positions at which the electron beam emerging from the linac was weakly convergent in both the vertical and horizontal planes. TSM is then used to determine by "computer experiments" the quadrupole focusing that provides the best settings for operation. This includes the requirement for matching as well as minimizing beam loss due to beam interception the small diameter apertures along the diagnostic chicane. Then the best "model solution" is found for the settings of the beam line quads. Finally, the setup is checked using these settings to transport the actual ebeam through the beam line. The required focusing for the focus at the IP is illustrated using ray optics in Fig. 6 which also maintains beam quality for driving the IR FEL. The corresponding beam envelope evolution implemented and recorded on-line during the experiment is also shown in Fig. 7. With sufficient data and feedback, the TSM can be improved and enhanced to run routinely independent of an operator in the future.

\section{FEL laser optimization}

\section{Feed-forward phase and amplitude stabilization}

The performance of rf linac driven free-electron laser oscillators is critically dependent on the amplitude and phase uniformity of the high-power microwave pulses used to drive the electron gun and linac. Amplitude nonuniformity contributes to an energy spread among the electron bunches and a loss of laser efficiency. Phase irregularities in the rf waveform result in variations in the arrival time of the electron bunches, which reduces laser gain due to a loss of synchronism between the electron bunches and the circulating optical pulses in the laser. For example, a phase 

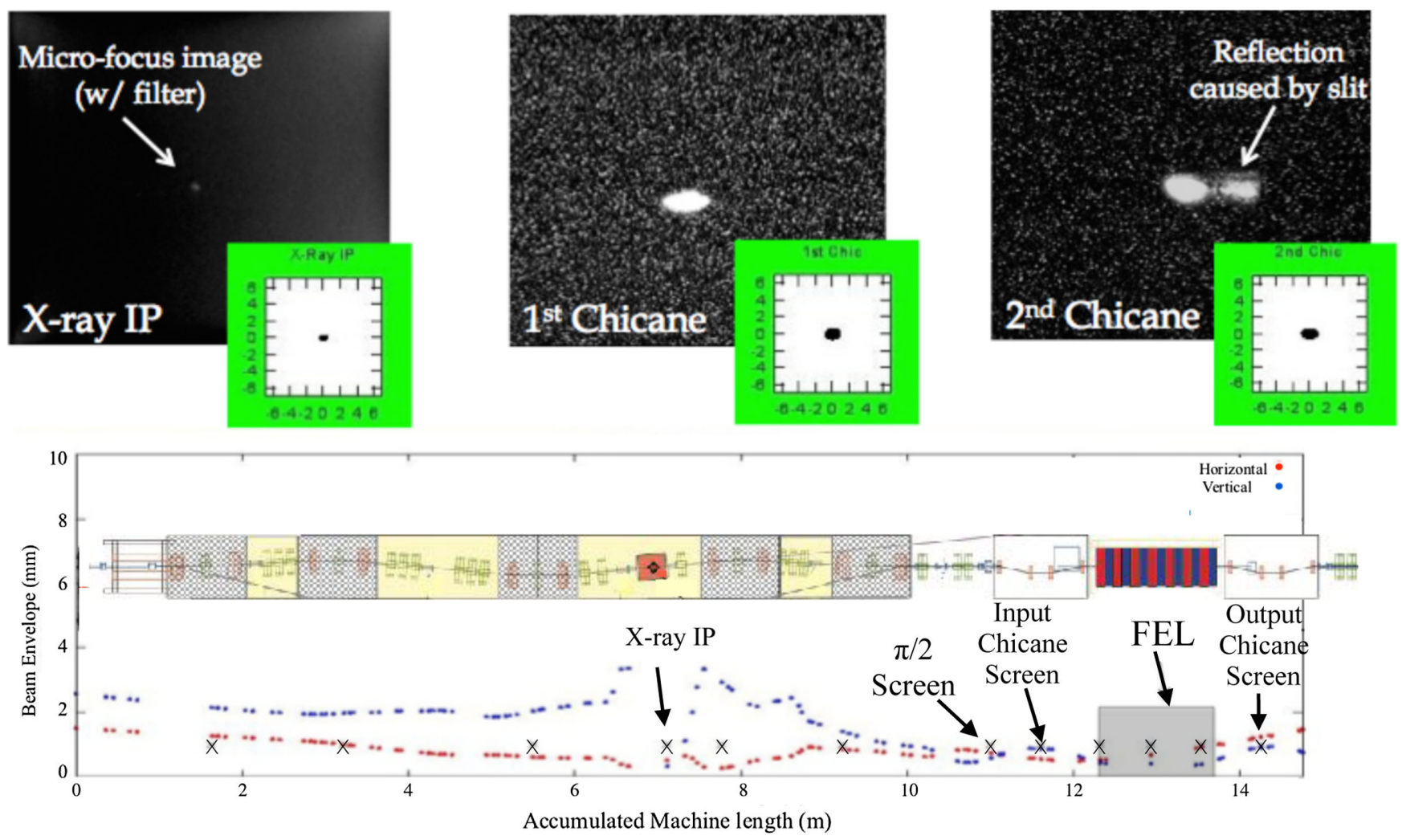

FIG. 7. Comparison of the implemented TSM for an optimum match and the measured beam profiles: The top images show a comparison between the OTR images of the beam at the X-ray IP, first chicane (FEL input chicane) and the second chicane (FEL output chicane) with the predicted beam profiles from the TSM. The lower section shows the predicted evolution of the electron horizontal (red) and vertical (blue) envelope size through the machine for an optimum match. "x" indicates the position of diagnostic screen. A quarter inch $\mathrm{SiC}$ mirror is used for imaging the electron beam at IP.

excursion of $1^{\circ}$ at $2856 \mathrm{MHz}$ delays the electron bunches by $1 \mathrm{ps}$, which is the full width of a typical optical pulse, leading to reduced laser gain and reduced laser power.

Electron bunch synchronization is of particular importance for the operation of optical storage cavity-based
inverse-Compton $\mathrm{x}$-ray sources, such as FELICIA. Incoming electron bunches must be synchronized to the circulation time of laser pulses in the storage cavity, and any loss of beam synchronism in the scattering region will lead to reduced x-ray flux [28].

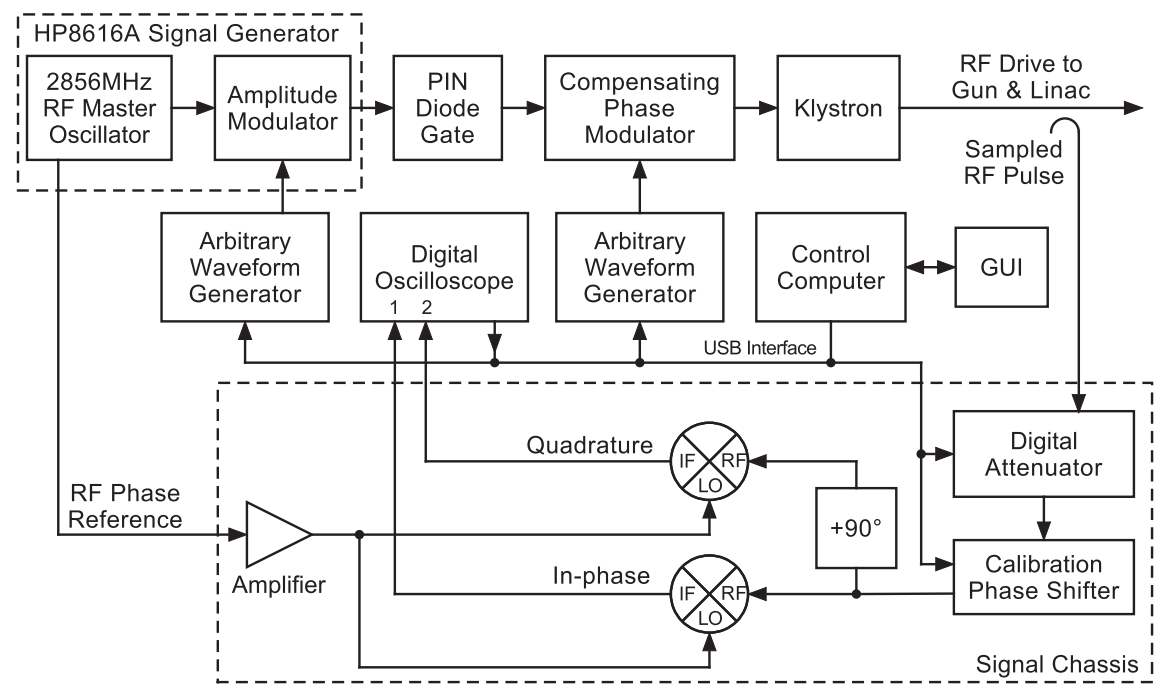

FIG. 8. Block diagram of the MkV feed-forward system. 


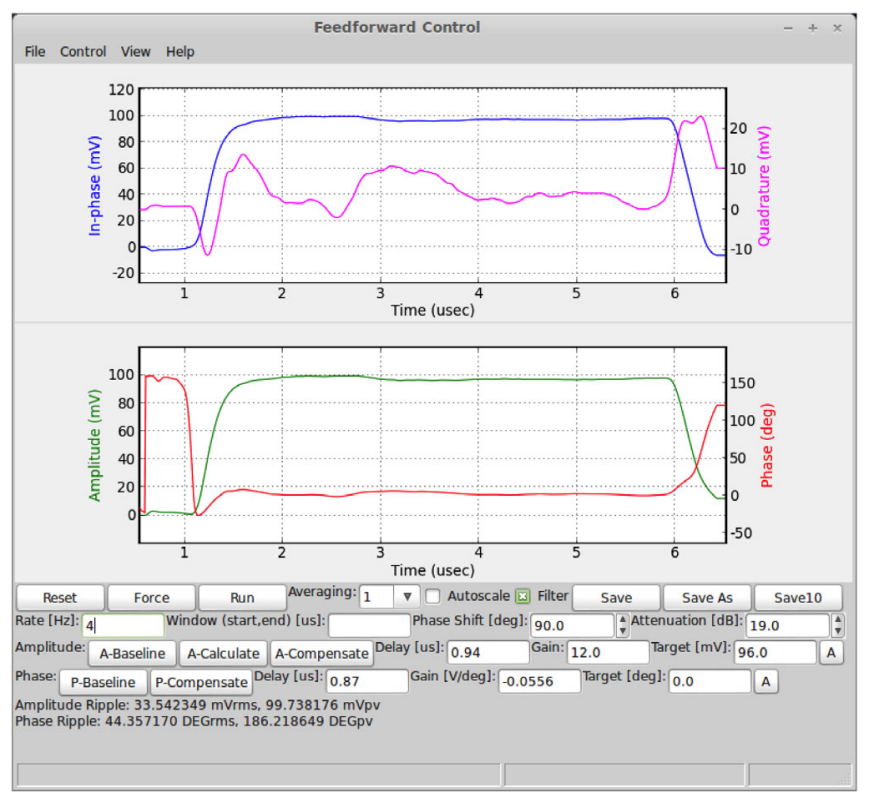

FIG. 9. Feed-forward GUI: The graphical user interface (GUI) for the feed-forward system. The upper plot shows the raw sampled in-phase and quadrature signals, while the bottom plot shows the reconstructed amplitude and phase. System commands are issued using the buttons at the bottom of the window.

An active feed-forward stabilization system has been successfully integrated into the low-level rf subsystem of the MkV FEL [28,29]. This system, depicted in Fig. 8, samples the high power if drive signal from a directional coupler at the input to the linac. The signal is quadrature demodulated and sampled with a digital oscilloscope. The feed-forward software, Fig. 9, generates compensation waveforms that are applied to an amplitude modulator and phase shifter in the low-level rf system for application to subsequent $\mathrm{rf}$ macropulses.

The system can be operated to iteratively refine the compensation waveform for optimal rf flatness. Figure 10 shows an $85 \%$ ripple reduction in the rf drive to the linac

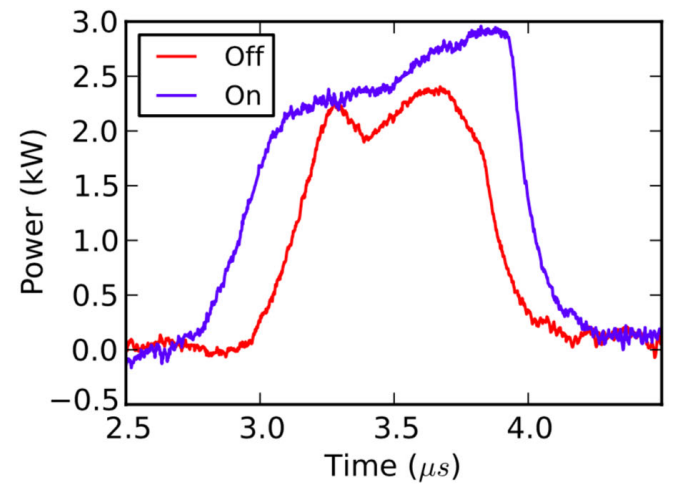

(a)
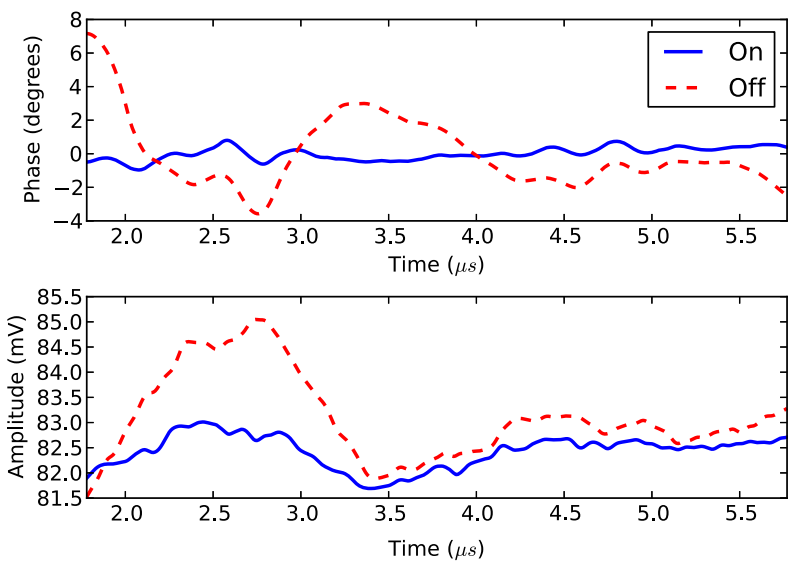

FIG. 10. Effect of compensation: Feed-forward phase and amplitude compensation result in an $85 \%$ rf power ripple reduction.

while the electron beam energy spread is reduced by $57 \%$. The effectiveness of the stabilization system are best observed in the laser power and spectrum (Fig. 11) where improved rf phase stability leads to a $40 \%$ increase in pulse length and a $20 \%$ decrease in laser linewidth. Note that this stabilization was accomplished using the current from the microwave gun to adjust the rf voltage, so the stabilization scheme intrinsically compensates for beam loading effects in the gun. The system is capable of providing some compensation of beam loading in the linac for the operational beam and rf parameters described here as well. Further capability of the system was not explored here, however, similar capabilities have been studied in depth for similar s-band SLAC type linacs [30].

The rf receiver portion of the feed-forward system also serves as a general purpose synchronous rf diagnostic tool for visualization of signals from rf pickups on BPMs and high speed optical detectors for laser diagnostics. This system was a critical tool for the synchronization of laser and electron pulses at the inverse-Compton interaction point [31].

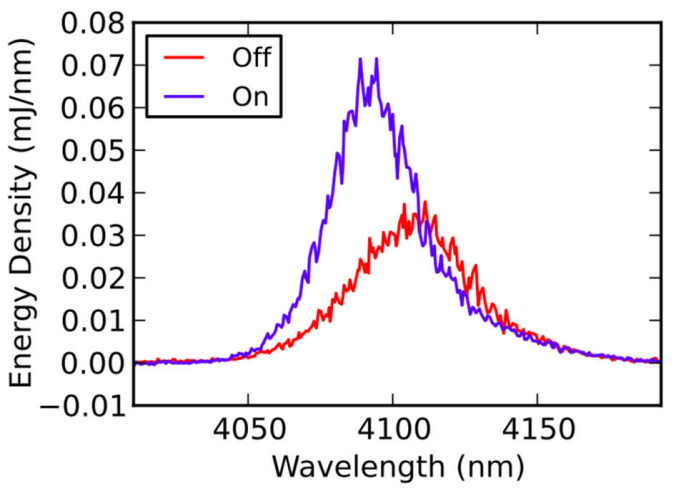

(b)

FIG. 11. Measured effect of the feed-forward system on laser performance. (a) The laser pulse power and (b) laser spectrum are computed as the integrals of the spectrogram plot in $\lambda$ and $t$ respectively. The compensation lengthens the pulse duration by $40 \%$, while the spectrum narrows by $20 \%$. 


\section{Phase-locked operation of the FEL}

The Compton backscattered photon flux is directly proportional to the incident laser intensity. To maximize the incident intensity, the FEL is operated in the phaselocked mode of operation [32-35] in which successive optical pulses in the gigahertz-rate, laser pulse train are forced to exhibit interpulse phase coherence, i.e., the same relative optical phase between each successive pair of pulses. This condition is achieved by employing a laser intracavity interferometer to optically couple successive pulses in the FEL resonator and thus enforce interpulse phase coherence during the amplification process. Since the coupling of successive pulses requires that the pulses in the two arms of the interferometer arrive simultaneously at the beam splitter, the phase-locked and non-phase-locked modes of operation are readily interchanged by delaying the arm of the interferometer to bring the pulses into and out of coincidence.

Phase locking of the FEL in the present project serves two important purposes. First, it increases the outcoupled laser power from the FEL by roughly a factor of 2 [36]. The MkV FEL employs Brewster plate output coupling in which one of the two reflective surfaces provides the outcoupled beam. Reflections from the second surface otherwise constitute a source of cavity loss. By using this second surface as the intracavity beam splitter to couple successive pulses in the FEL, these second surface reflections are eliminated due to destructive interference as the interpulse phase coherence develops in the pulse train. The reduction of cavity loss by a factor of 2 leads to an increase in outcoupled power by roughly the same factor, as well as a decrease in the turn-on time and corresponding increase in the optical macropulse duration. Measurements of the outcoupled laser macropulse power with and without phase locking are shown in Fig. 12. In this figure, phase locking was induced among every third laser pulse, yielding 13 interleaved sets of phaselocked optical pulses within the laser cavity.

The second and more fundamental purpose served by phase locking related to future high-brightness $\mathrm{x}$-ray generation is in the efficient pumping of the ICS optical

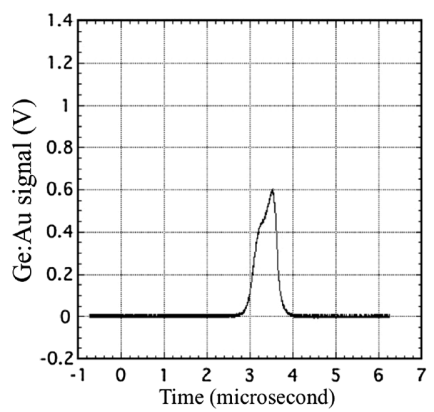

(a)

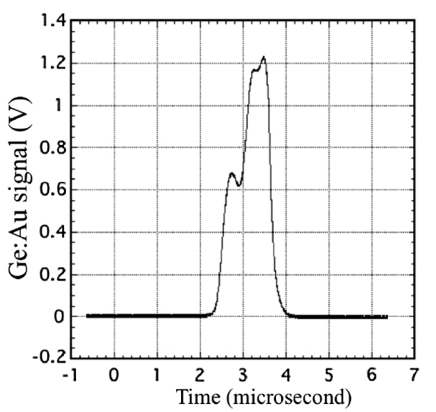

(b)
FIG. 12. Measured laser macropulse: outcoupled laser power in the (a) non-phase-locked and (b) phase-locked modes of operation. storage cavity, which is designed to produce an optical undulator at the interaction point with the electron beam. The design and status of this development are described below in Sec. IV B. The necessity for phase locking arises from the fact that the optimized storage cavity is significantly shorter than the laser resonator. As a result, successive laser pulses having random optical phases would otherwise prohibit the coherent buildup of optical power in the storage cavity. The coupling of every third optical pulse in the FEL resonator yields more efficient phase locking than the coupling of adjacent optical pulses. Thus, the number of circulating optical pulses in the storage cavity is designed to be a multiple of 3 . In the current design of the full-up system, the storage cavity contains nine circulating optical pulses, compared to 39 in the FEL resonator.

The optical transport system from the FEL to the IP employs a long run 3-inch diameter evacuated pipe to deliver the laser to the IP optical bench with minimum atmospheric absorption of the IR light, followed by a shorter path in ambient air allowing the insertion of easily accessible IR optical components that serve the following purposes: (i) collimating the laser beam, (ii) inserting a precision optical trombone delay line to adjust the synchronism of the optical pulses and electron bunches, and (iii) a final steering mirror and focusing lens to deliver and align a $\mathrm{TEM}_{00}$ Gaussian mode of $30 \mu \mathrm{m}$ radius to the IP.

\section{IP scattering chamber and diagnostics}

At the heart of FELICIA is a custom-built vacuum chamber surrounding the inverse-Compton scattering interaction point and supporting all the optics and diagnostics necessary to collide the focused beams. While preliminary experiments have focused on the optimization of a singlepass interaction between the laser and ebeam this vacuum chamber is readily extensible to house the mirrors of an optical storage cavity. Table I summarizes the design specifications for single-pass operation of the inverseCompton x-ray source and establishes the design constraints for the vacuum chamber.

Figure 13 shows an overview of the x-ray scattering chamber and associated diagnostics. At the top, the laser

TABLE I. Single pass x-ray scattering experiment specifications.

\begin{tabular}{lcc}
\hline \hline Wavelength & $\lambda$ & $3 \mu \mathrm{m}$ \\
IP Rayleigh range & $z_{R}$ & $1 \mathrm{~mm}$ \\
IP spot size & $w_{0}$ & $31 \mu \mathrm{m}$ \\
Beam crossing angle & $\alpha$ & $5.75^{\circ}$ \\
IP divergence angle & $\theta_{\frac{1}{2}}$ & $1.77^{\circ}$ \\
X-ray photon energy & & $10.4 \mathrm{keV}$ \\
Ebeam energy & & $40 \mathrm{MeV}$ \\
Ebeam emittance & $\epsilon_{n}$ & $8 \pi \mathrm{mm} \mathrm{mrad}$ \\
Micropulse duration & $\tau_{\mu}$ & $1 \mathrm{ps}$ \\
\hline \hline
\end{tabular}



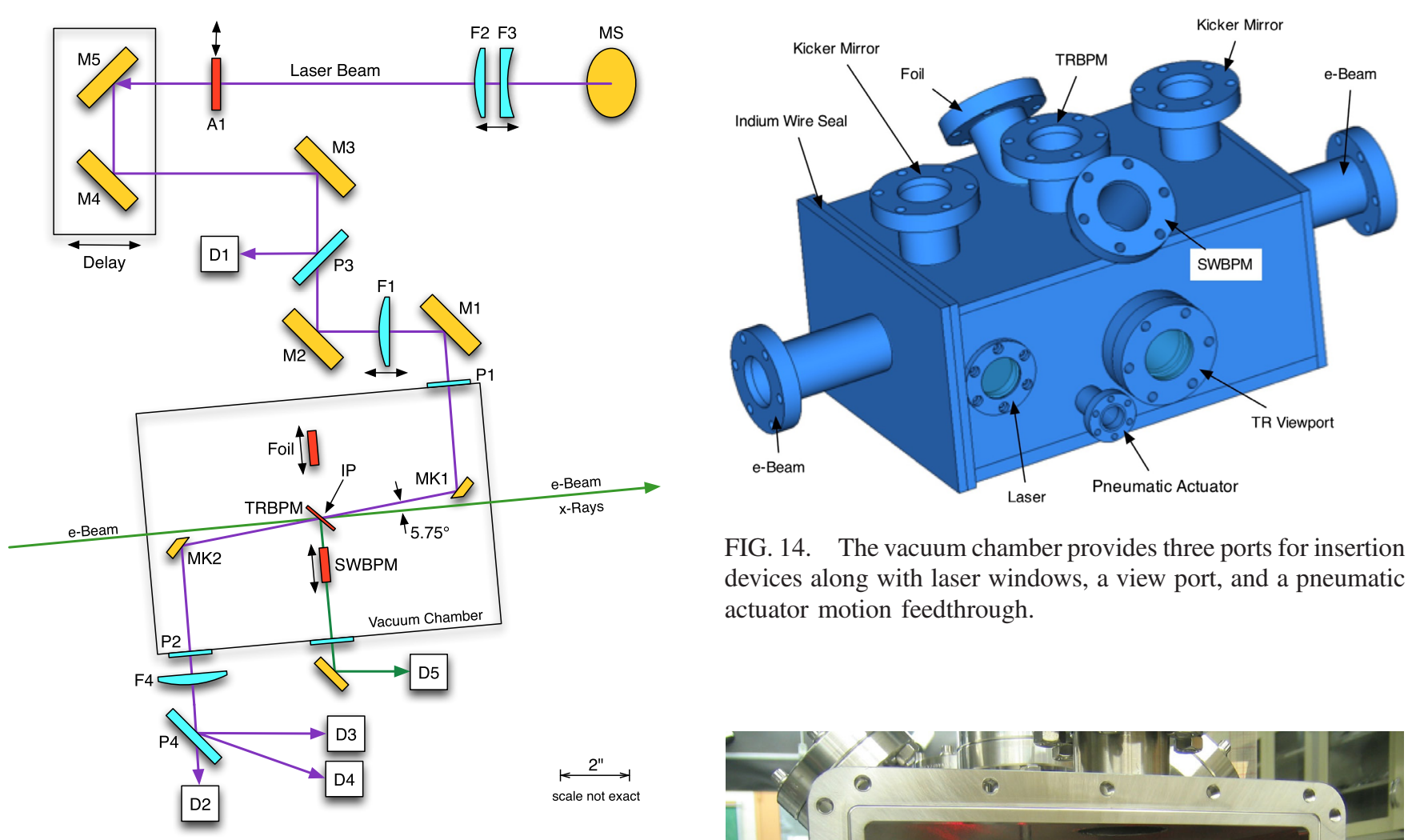

FIG. 14. The vacuum chamber provides three ports for insertion devices along with laser windows, a view port, and a pneumatic actuator motion feedthrough.

FIG. 13. The optics for the single-pass x-ray system delivers the focused laser beam to the interaction point. The input beam incident on mirror MS is collimated with the F2/F3 telescope. An optical delay line is used to adjust the arrival time of pulses at the IP and lens F1 focuses the beam for scattering. Steering of the beam at the IP is accomplished with motor-driven mirror M1. The output side of the vacuum chamber provides a number of beam diagnostics.

beam is directed from FEL, focused, delayed, and aligned to the IP. Small retractable kicker mirrors (MK1 and MK2) in the vacuum chamber provide a small beam crossing angle to eliminate the need for the electron beam to couple though a hole in the mirrors. The beam crossing angle was chosen as close as practical to $180^{\circ}$ to maximize the scattered x-ray energy and beam intersection volume to relax the temporal synchronization requirements for initial alignment. The used beam exits the chamber on the reverse side and is available for laser beam diagnostics such as power and beam profile. The chamber is installed in a drift section with focusing quadrupole magnets on either end. Three ports provide access to the interaction point for diagnostic devices including a scanning wire beam profilometer, bremsstrahlung foil target, and optical transition radiation screen. A side viewing port allows transition radiation to be imaged on a TV camera, in this case a radiation-resistant vidicon tube, similar to the ones used for all the OTR screens because of the proximity of the electron beam. The vacuum chamber itself (Fig. 14) is constructed from 0.25 -inch stainless steel plate assembled and welded

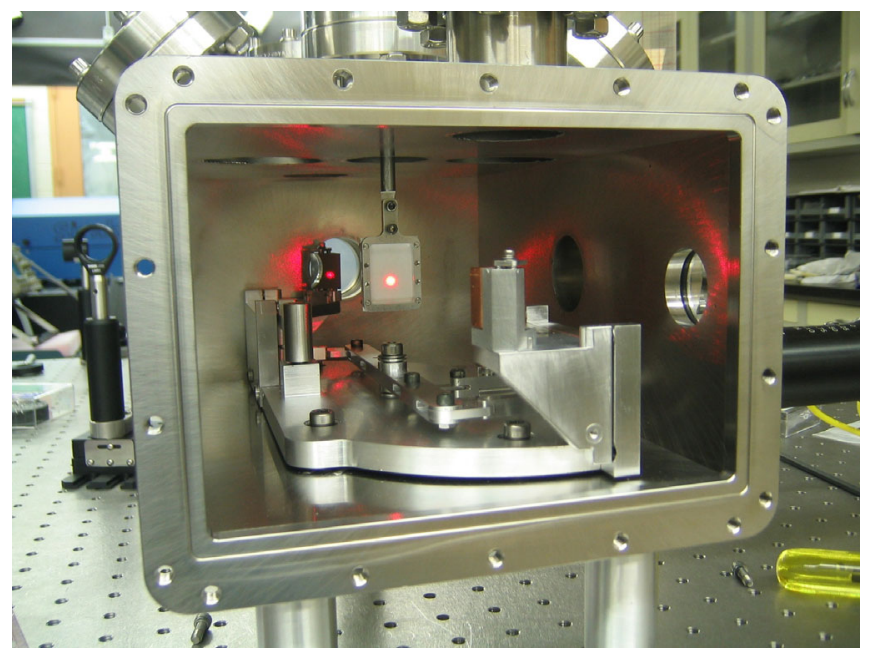

FIG. 15. The interior of the assembled vacuum chamber is shown with the upstream flange removed. The alignment $\mathrm{HeNe}$ beam can be seen on the far kicker mirror and on the paper alignment screen.

in-house. The internal mirror retraction system shown in Fig. 15 consists of an aluminum breadboard kinematically centered in the vacuum chamber and actuated pneumatically with beam position repeatability better than $2 \mu \mathrm{m}$. The mirrors may be retracted away from the electron beam axis to protect the mirror surfaces from beam damage during alignment and focusing operations as well as nonICS operation of the accelerator.

The fully integrated interaction point is shown in Fig. 16 with all diagnostic devices and optics. Also visible are the focusing quadrupole magnets and a strip-line beam position monitor used to focus and center the electron beam. 


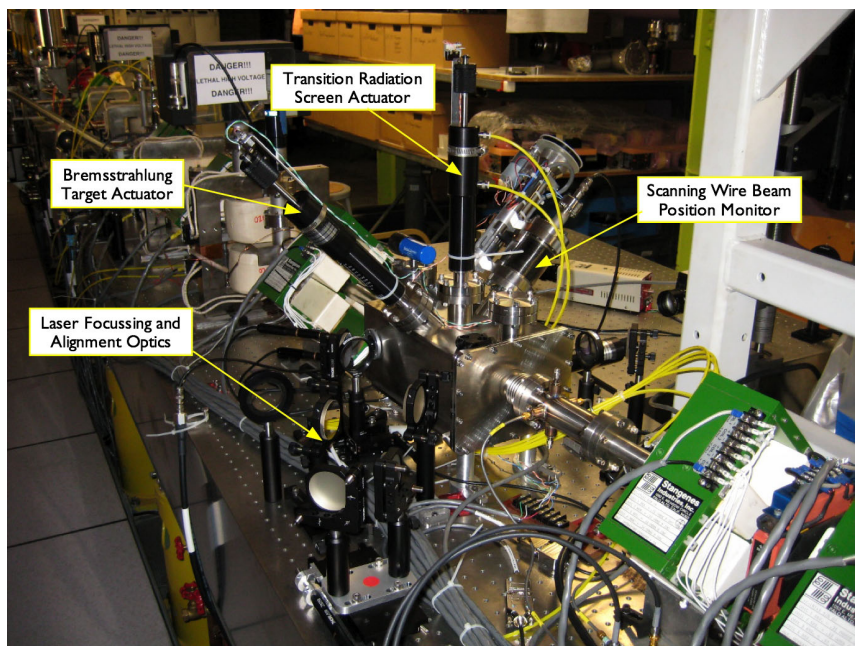

FIG. 16. The x-ray scattering chamber is installed in the MkV diagnostic chicane beam line. Three insertable devices and laser transport optics are also installed.

\section{Scanning-wire beam profilometer}

Coalignment of the microfocused electron and laser beams is a challenging requirement of any inverseCompton scattering system. With high intensities, and spot sizes as small as $30 \mu \mathrm{m}$, it is not possible to align these beams without special diagnostic tools. Since the resolution of available BPMs and OTR screens are not adequate for FELICIA, a custom scanning wire system has been developed and described in detail in [37].

Wire scanners are commonly employed on accelerator beam lines as alignment aides. The "flying wire" type scanners, such as those used at CERN, are too large for use in the space allocated on the $\mathrm{MkV}$ beam line at $\mathrm{UH}$ and are incompatible with the bunch structure of our accelerator. The x-ray interaction point is shared by two other insertable diagnostic devices in a crowded vacuum chamber, also

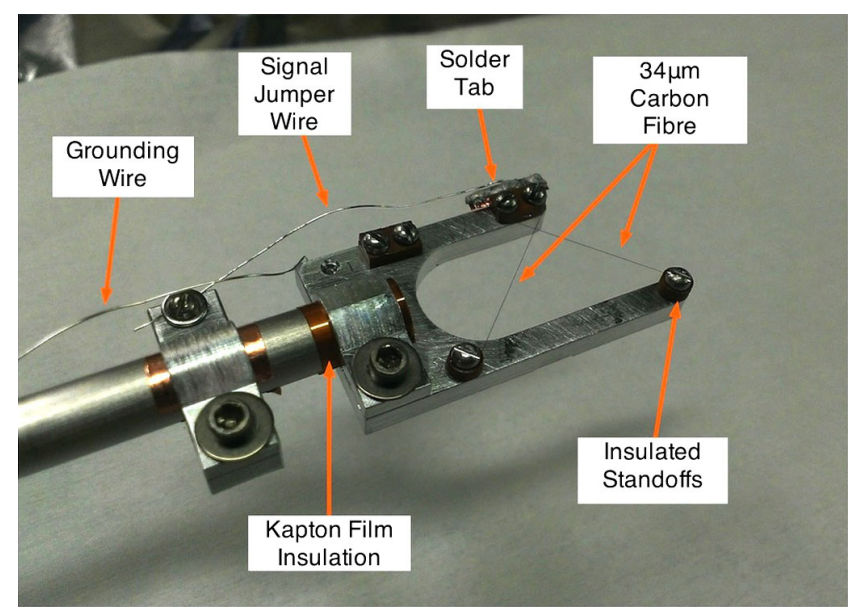

FIG. 17. The wire scanner fork electrically isolates the carbon fiber from the grounded fork. Fibers are soldered to the signal lead and clamped at the other end.

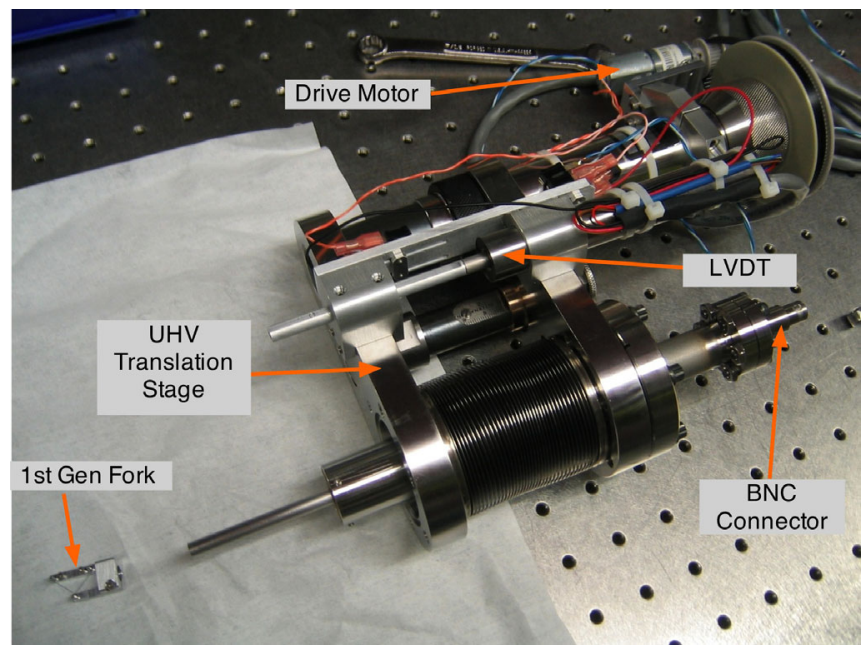

FIG. 18. Beam profiler drive assembly with motor and LVDT with an early prototype fork. The support rod conducts the signal to the vacuum feedthrough on the far end.

housing the x-ray interaction point laser optics. The wire scanner described here is based on the designs used at NBS-LANL [38] and the SLC [39] and adapted to the constraints of our beam line configuration. This system also includes the capability to resolve the temporal evolution of the electron beam profile over the macropulse duration (approximately $4 \mu \mathrm{s}$ ).

The wire scanner head shown in Fig. 17 consists of two $34 \mu$ m-diameter graphite fibers stretched across the $12.3 \mathrm{~mm}$ gap in an aluminum fork. The wires are oriented such that when the scanner insertion axis is inclined $45^{\circ}$ above the beam plane, the two wires are oriented horizontally and vertically. In this way a single axis of motion allows the beam to be scanned in both axes. The secondary emission current from the wire is conducted via the scanner shaft to a vacuum feedthrough on the assembly shown in Fig. 18. The wire scanner system illustrated in Fig. 19 consists of motion control and data acquisition electronics integrated with a graphical user application. A pyroelectric detector viewing the transmitted laser beam measures the occlusion by the wire.

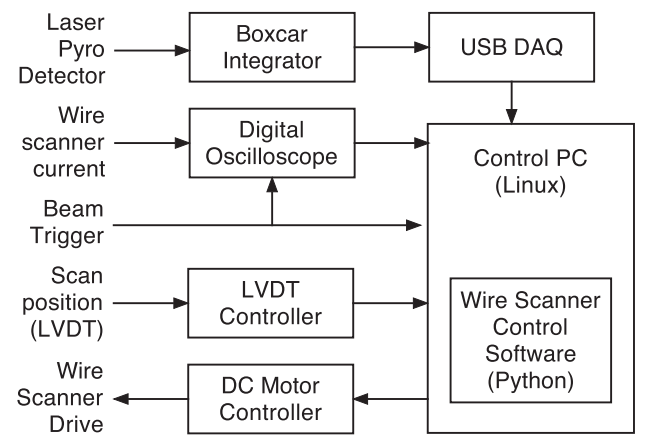

FIG. 19. A PC acquires beam current, laser intensity, and scan position data and controls the drive motor. 


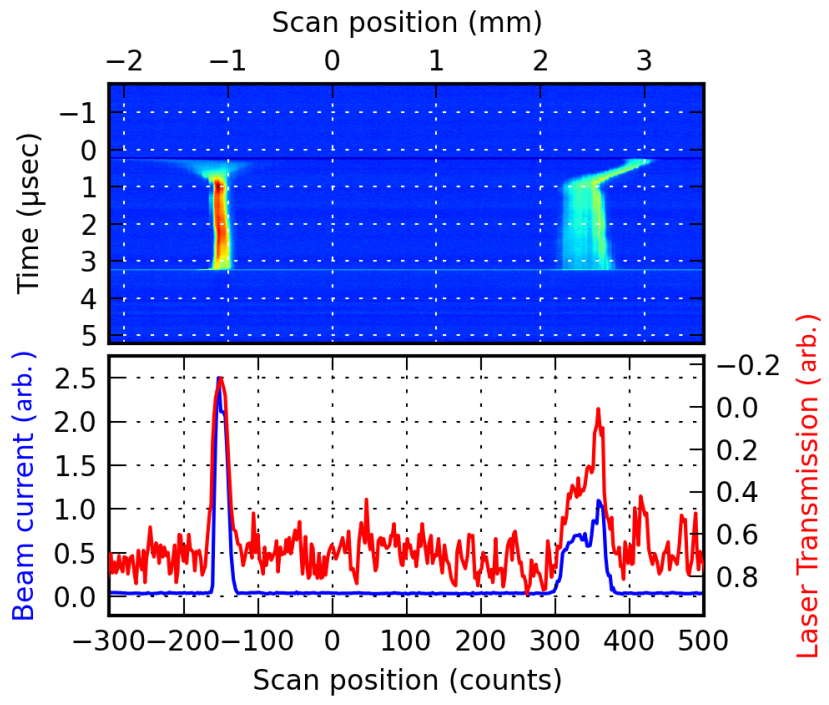

FIG. 20. Secondary emission current as a function of position and time within the macropulse. The lower plot compares the integrated current from 2 to $3 \mu$ s with the laser signal (axis inverted). This scan includes both the horizontal (right) and vertical (left) profiles.

Figure 20 shows an electron beam focused to $w_{x}$, $w_{y}=350 \mu \mathrm{m}, 115 \mu \mathrm{m}$ FWHM, where the left feature is the time evolution of the vertical beam profile and the right represents the horizontal. In this scan the laser is well aligned to the electron beam resulting in suppression of laser operation while the beam is intercepted by the wire.

Figure 21 shows an example where the laser and the electron beam are misaligned. This horizontal scan (vertical wire only) gives an electron beam width of $175 \mu \mathrm{m}$ and a laser beam width of $143 \mu \mathrm{m}$ with a beam separation of

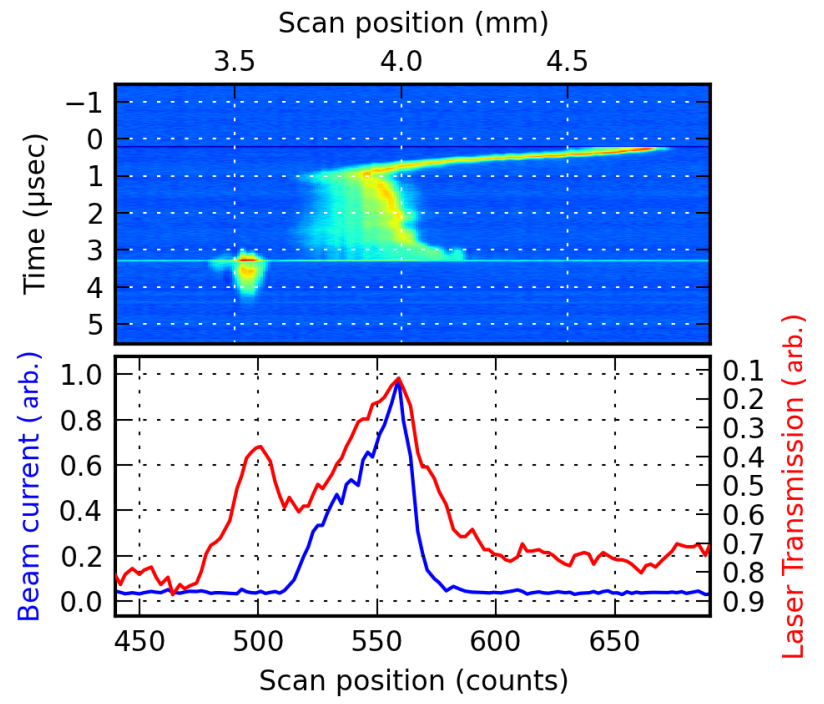

FIG. 21. Horizontal axis wire scan showing the laser displaced from the ebeam position. Interception of the ebeam by the wire inhibits lasing and results in a second coaligned peak.
$419 \mu \mathrm{m}$, correctable with a motorized mirror. The laser was intentionally defocused at the interaction point for this scan to preclude wire damage during these early experiments. Figure 21 also illustrates the time resolved nature of this wire scanner system. The trajectory in this image indicates that the beam's horizontal position slews nearly a mm over the $4 \mu \mathrm{s}$ macropulse. The large slew in the first microsecond is an inevitable consequence of beam loading in the linear accelerator and is typically ignored for experimental purposes. The remainder of the macropulse, however, also shows a position slew that is a consequence of energy slew in the beam. Characterization and mitigation of this energy/ position slew in the beam are of critical importance to operation of the free-electron laser and for beam alignment of the inverse-Compton scattering interaction. A naive integration of the wire current or an optical transition radiation image would significantly overestimate the instantaneous beam size as well as produce an ambiguity in the centroid position during the time interval of interest for scattering. Even with significant transverse evolution in the beam, the instantaneous size and position can be precisely measured by the time-resolved diagnostic.

The custom wire-scanner design satisfies the tight space restrictions imposed by the inverse-Compton x-ray interaction point and the use of a commercially available linear vacuum translator significantly reduces the engineering time and cost of the system. A $34 \mu \mathrm{m}$ carbon fiber has proven a suitable material for scans of a sub- $100 \mu \mathrm{m}$ microfocused electron beam operated at $40 \mathrm{MeV}$ with $150 \mathrm{~mA}$ average current over $4 \mu$ s macropulses with a scan resolution of $7 \mu \mathrm{m}$ and scan repeatability of better than $10 \mu \mathrm{m}$.

\section{Temporal synchronization of the $\boldsymbol{x}$-ray interaction point}

Since the same electron beam is used to drive both the $\mathrm{x}$-ray scattering interaction and the FEL, the fixed phase relationship between the $\mathrm{GHz}$ rep-rate electron microbunches and optical pulses reduces temporal synchronization to a matter of determining a fixed delay. To optimize $\mathrm{x}$-ray luminosity it is important that the arrival times of the micropulses at the interaction point are synchronized. The interaction should be confined to the $2 \mathrm{~mm}$ long confocal region of the laser at the designated interaction point and the 3 ps micropulse duration requires picosecond level synchronization. Given the 350 ps interpulse spacing corresponding to the $2.856 \mathrm{GHz}$ repetition rate, this synchronization requirement corresponds to an $\mathrm{rf}$ phase synchronization of less than $1^{\circ}$.

Measurement of the micropulse arrival times at the interaction point is confounded by several factors: high radiation backgrounds, the broad bandwidth of optical transition radiation, low OTR intensity at $3000 \mathrm{~nm}$, the detection of low level IR light, detector bandwidth, etc. 


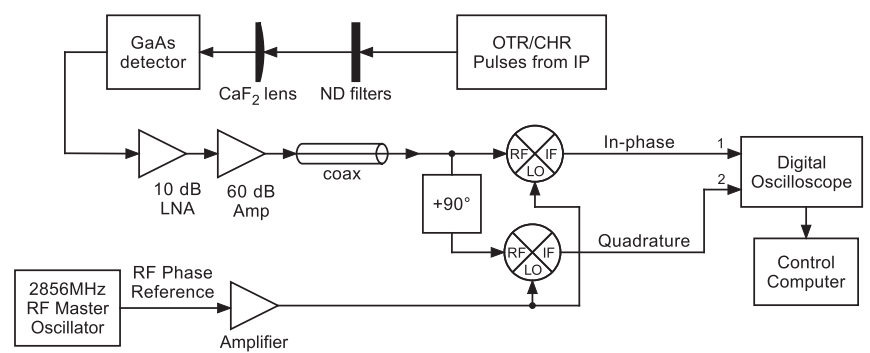

FIG. 22. OTR and CHR signals detected by a fast GaAs photodiode are amplified and then demodulated and digitized by the complex receiver portion of the feed-forward amplitude and phase compensation system. The quadrature and in-phase signal waveforms are used to reconstruct the amplitude and phase of the $2.856 \mathrm{GHz}$ signals.

The approach for FELICIA compares the rf phase of the visible OTR from a copper mirror inserted into the electron beam with the visible coherent harmonic radiation (CHR) generated by the FEL as previously reported in [31]. Since the CHR is coaligned with the fundamental laser light, it is useful as both a transverse alignment aid and a temporal reference for synchronization. A water cell was used as an IR blocking filter to pass only visible spontaneous radiation from the undulator. This allows a single visible rf detector measure both OTR and laser pulse phase. Since the rf phase of the $\mathrm{GHz}$ repetition rate pulse trains is being compared, not the optimal phase of the radiation, the effects of dispersion on pulse phase are negligible.

The rf stabilization system described in Sec. II C 1is used as a precision rf phase comparator for pulse synchronization. Accepting the system's master oscillator as an absolute phase reference we can make independent but precisely calibrated measurements of the relative phases of the OTR and CHR light. A simplified block diagram of this phase measurement system is shown in Fig. 22.

A copper mirror oriented at $45^{\circ}$ is inserted into the electron beam path for imaging the electron beam using OTR. Since the laser beam is counterpropagating the same mirror cannot be used for extraction of the laser beam. Moreover, the unperturbed electron beam is required downstream of the IP for operation of the FEL. For the purpose of synchronization, a small silvered silicon mirror is mounted below the copper OTR mirror and inserted using the same pneumatic actuator but with a different insertion depth. This geometry is shown in Fig. 23 with the mirror position offset from the electron beam to provide a clearance of $5 \mathrm{~mm}$ when inserted (allowing for unimpeded FEL operation). It is aligned such that deflection of the laser beam onto the mirror by $\alpha=1.75^{\circ}$ results in a CHR reflection coaligned to the axis of the OTR light. A correction is applied to the measured laser phase to account for the path length difference of $d=6.78 \mathrm{~mm}$ associated with the offset path.

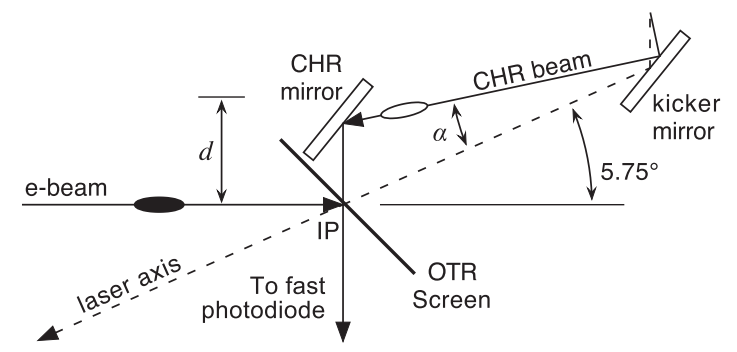

FIG. 23. A small mirror can be installed offset from the electron beam in place of the OTR screen. This mirror allows the CHR light from the FEL to be coaligned with the OTR light from the ebeam without obstructing the e-beam. The CHR pulses travel an additional distance $d$ relative to the laser beam when steered directly at the IP.

The relative rf phase of the OTR and CHR light detected with the fast photodiode is shown in Fig. 24. The optical path delay is varied by translation of a optical trombone and shows the expected linear relationship with the rf phase. The phase shift is extrapolated to zero the phase difference between the OTR and CHR pulses including the effect of path length differences due to mirror offset and optical attenuators.

This procedure allows the optical trombone to be adjusted to an uncertainty of less than $2^{\circ}$, thus reducing the delay scan range for empirical optimization of $x$-ray production from 52 to $0.3 \mathrm{~mm}$.
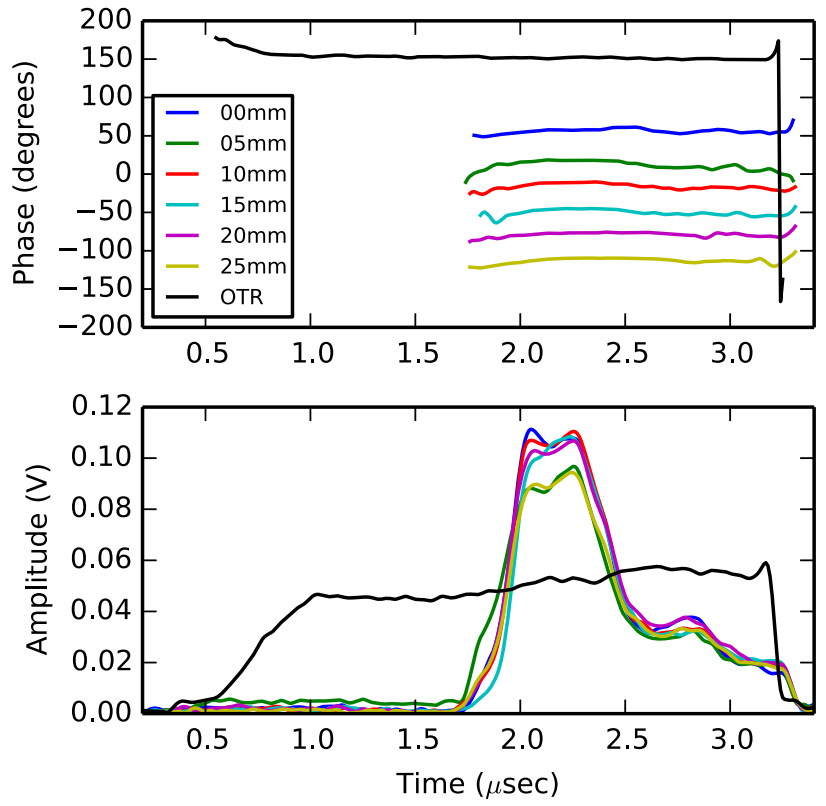

FIG. 24. Phase and amplitude of the $2856 \mathrm{MHz}$ component of the OTR light compared to the CHR light at various trombone positions. The rf background pedestal has been subtracted from these data and the phase for signals with an amplitude (relative to background) less than $6 \mathrm{mV}$ is excluded. Each trace shown is the average of ten subsequent pulses with the background subtracted from each. 


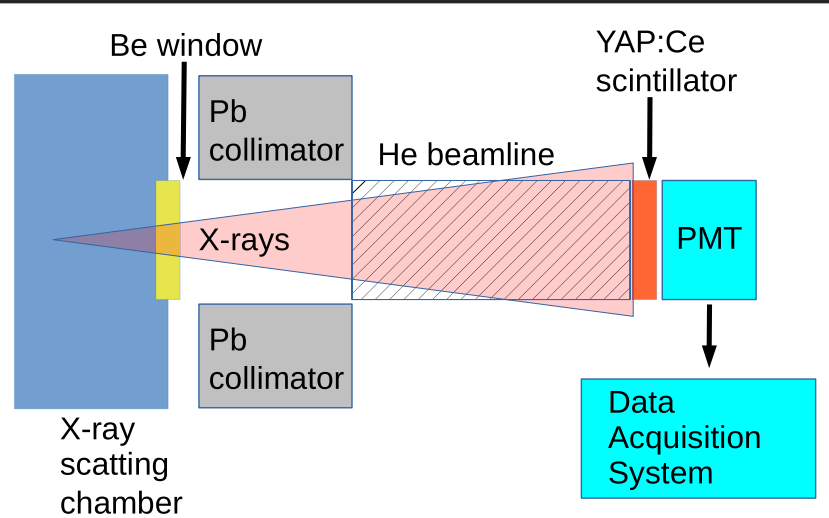

FIG. 25. X-ray transportation and detector system.

\section{E. X-ray transport and detection system}

\section{X-ray beam line components}

The $\mathrm{x}$-ray transport beam line and detection system consists of the following components: an $\mathrm{x}$-ray scattering chamber, a beryllium window, a lead collimator, a helium purged beam pipe, a yttrium aluminum perovskite activated by cerium (YAP:Ce) scintillator, and a Hamamatsu 6199 photomultiplier tube (PMT). Data acquisition is performed using a Molectron dual gate generator for setting the gate to the last microsecond of the pulse on an oscilloscope, a Model 4121B boxcar averager, and a Hewlett Packard 7035B x-y plotter (Figs. 25 and 26). The $0.25 \mathrm{~mm}$ thick, $15 \mathrm{~mm}$ diameter beryllium window transmits $\mathrm{x}$-rays with energy between 3 and $12 \mathrm{keV}$ with $>90 \%$ efficiency, decreasing outside this range (see Fig. 25), so any radiation of substantially lower/higher energy (due to the electron beam scraping the vacuum chamber walls) is blocked. The attenuation coefficient of helium is 153 times less than air,

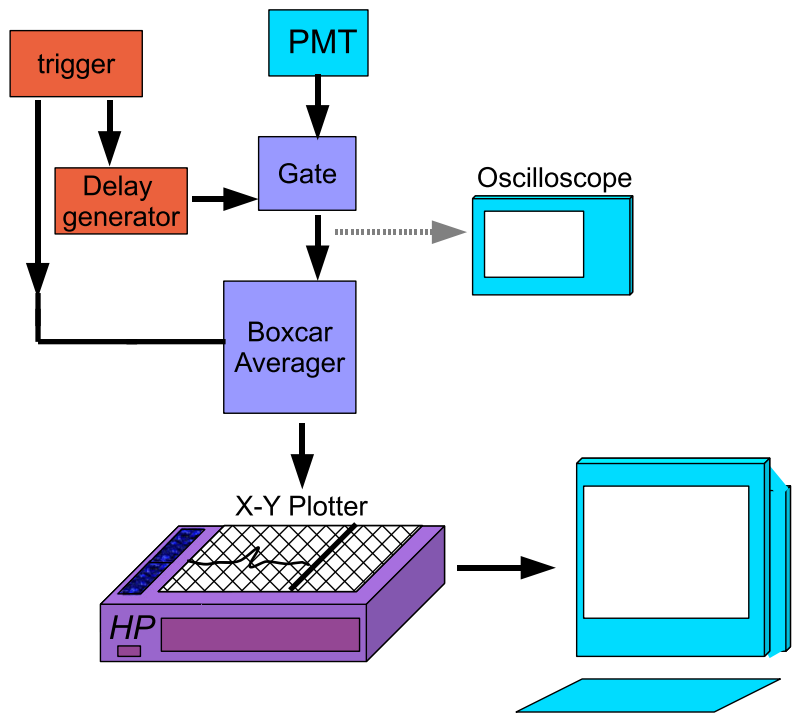

FIG. 26. X-ray data acquisition setup. The interval for recording the signal is set by means of the delay and gate on the scope. Then the signal is sent through the boxcar averager and recorded by the $x-y$ plotter.

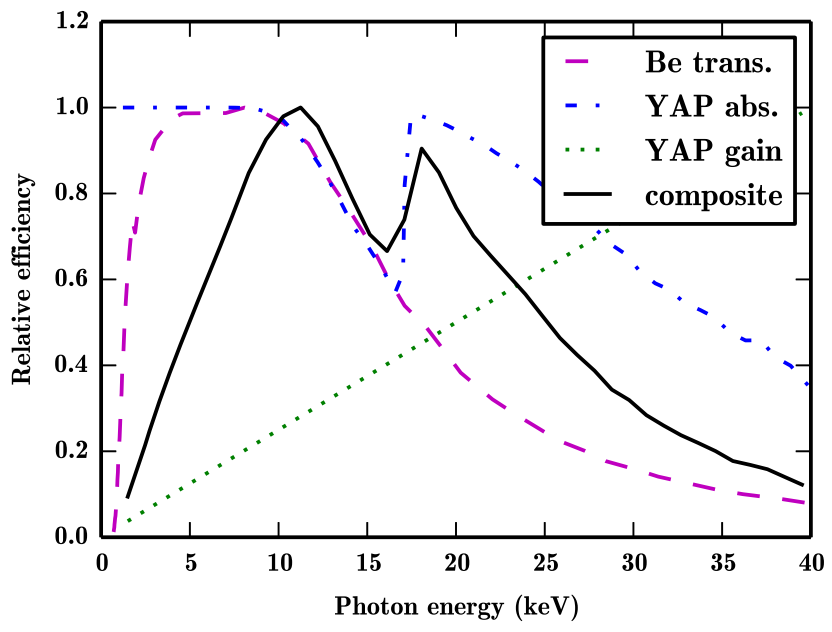

FIG. 27. The beryllium transmission, YAP:Ce absorption and photon gain, and composite x-ray transportation and detector system efficiency as a function of energy.

so a purged beam line was used to transport the x-rays from the scattering chamber to the detector (a distance of approximately 10 meters). The YAP:Ce absorbs all incident $10 \mathrm{keV}$ photons (but is transparent to higher energy photons) and converts them with high gain to $370 \mathrm{~nm}$ (blue) photons for detection by the PMT. Figure 27 shows the transmission function of the transport and detection system as a function of energy. An oscilloscope and personal computer are used to digitize the measurements by the data acquisition setup.

\section{System calibration}

Calibration of the system was checked by colliding the electron beam into a beryllium foil (separate from the window) and matching the expected yield of x-rays in the energy passband to the signal from the detection system. A boxcar integrator was used to measure average flux with the gate aligned to sample the final microsecond of the macropulse. The output of the boxcar integrator was recorded by a Hewlett Packard $\mathrm{x}-\mathrm{y}$ plotter and digitized (Fig. 26).

\section{Radiation background}

We discovered that even when the beamstop was stopping the electron beam before entering the linac, a significant background signal was detected. A careful investigation revealed that this signal was due to the production of neutrons in the lead shielding material near the IP. These neutrons also produced fluorescence in the YAP:Ce and PMT glass envelope which also contributed to the background signal. Three different measures were taken to reduce the amplitudes of the long initial and trailing edge of these pulses. First, we replaced the lead shielding blocks with aluminum blocks consisting of the 


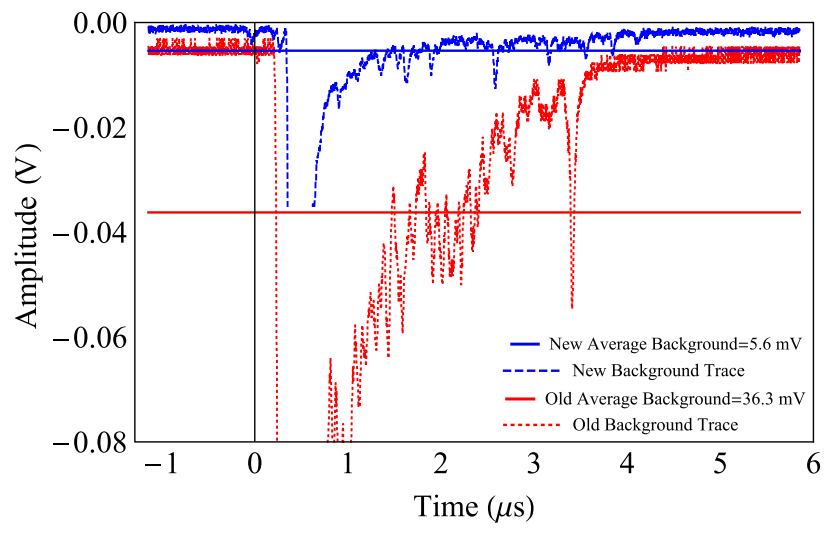

FIG. 28. Plot of background (without beamstop in) comparison (dotted red) before, (dashed blue) after installing aluminum blocks, thick polyethylene boards, and borated polyethylene. The solid lines show that the average background was reduced from 36.3 to $5.6 \mathrm{mV}$.

same number of radiation lengths. Second, we installed a series of one-inch thick polyethylene boards around the beam pipes to reduce the energy of the remaining neutrons before they could induce fluorescence in the glass. Third, we installed borated polyethylene $(5 \%$ borated-green color) squares with only a small aperture for $x$-rays between the steel frame and coils of the two quadrupoles before the end of the $\mathrm{x}$-ray pipes to provide a perfect path for the $10 \mathrm{keV} x$-rays. By these measures, we were able to reduce significantly and almost eliminate the described background radiation signal as shown in Fig. 28.

\section{INITIAL RESULT FROM FELICIA}

\section{A. Expected x-ray flux}

Using the electron beam and laser beam parameters in Table II, Eqs. (1) and (2) from [40], we predicted the total flux into all angles and the brilliance of the source (listed in Table II):

$$
\begin{gathered}
F=\sigma_{\mathrm{T}} \frac{N_{\mathrm{e}} N_{\mathrm{l}}}{2 \pi\left(\sigma_{\mathrm{e}}^{2}+\sigma_{1}^{2}\right)} f_{\text {ave }} \\
B=1.5 \times 10^{-3} \frac{F \gamma^{2}}{(2 \pi)^{2} \epsilon_{n, x} \epsilon_{n, y}} .
\end{gathered}
$$

Here $\sigma_{T}=8 \pi r_{\mathrm{e}}^{2} / 3$ is the Thomson cross section, $N_{\mathrm{e}}$ and $N_{1}$ are the number of electrons and photons in each pulse, $f_{\text {ave }}$ is the repetition rate and $\epsilon_{n,(x, y)}$ is the normalized emittance.

\section{B. Measured x-ray flux}

To check these predicted values, we utilize the in-house developed tools we described in the earlier section of this paper. Once electron beam focal and dispersion
TABLE II. Current design and experimental operating parameters for FELICIA. The flux and brilliance are calculated from the

\begin{tabular}{|c|c|c|}
\hline Symbols and parameters & Design & Experiment \\
\hline \multicolumn{3}{|l|}{ rf/electron pulses } \\
\hline$f_{r f}$ rf frequency $(\mathrm{GHz})$ & 2.856 & 2.856 \\
\hline$I_{m p}$ macropulse current $(\mathrm{mA})$ & 170 & 143 \\
\hline$\tau_{m p}$ macropulse duration $(\mu \mathrm{s})$ & 5 & 5 \\
\hline$f_{m p}$ macropulse frequency $(\mathrm{Hz})$ & 100 & 4 \\
\hline$Q_{\mu p}$ micropulse charge $(\mathrm{pC})$ & 60 & 50 \\
\hline$\tau_{\mu p}$ micropulse duration (ps) & 2 & $1-2$ \\
\hline $\mathcal{E}_{e}$ electron beam energy $(\mathrm{MeV})$ & 40 & 42.2 \\
\hline $\begin{array}{l}\epsilon_{n} \text { normalized emittance } \\
\quad(\pi \text {-mm-mrad })\end{array}$ & 8 & 25 \\
\hline \multicolumn{3}{|l|}{ Laser pulses } \\
\hline$\lambda_{0}$ laser wavelength $(\mu \mathrm{m})$ & 3 & 3.1 \\
\hline $\mathcal{P}_{l}$ laser power $(\mathrm{MW})$ & 2.6 & 0.3 \\
\hline \multicolumn{3}{|l|}{ Interaction point } \\
\hline$w_{0}$ laser beam waist $(\mu \mathrm{m})$ & 30 & 30 \\
\hline rms electron beam size $(\mu \mathrm{m})$ & 30 & $53(42 \times 64)$ \\
\hline $\mathcal{F}$ storage cavity finesse & 2000 & $\cdots$ \\
\hline $\mathcal{P}_{s}$ stored optical power $(\mathrm{GW})$ & 2.6 & $\cdots$ \\
\hline \multicolumn{3}{|l|}{ Scattered $\mathrm{x}$-rays } \\
\hline $\mathcal{E}_{\gamma}$ x-ray energy $(\mathrm{keV})$ & 10 & 10.9 \\
\hline$\lambda_{\gamma}$ x-ray wavelength $(\AA)$ & 1.2 & 1.1 \\
\hline $\mathcal{F}_{\gamma}$ total average $\mathrm{x}$-ray flux $(\mathrm{ph} / \mathrm{s})$ & $2.1 \times 10^{11}$ & $3.0 \times 10^{5}$ \\
\hline $\begin{array}{l}\theta=1 / \gamma \mathrm{x} \text {-ray divergence angle } \\
(\mathrm{mrad})\end{array}$ & 12.6 & 12.1 \\
\hline $\mathcal{B}_{\text {ave }}$ average brilliance ${ }^{\mathrm{a}}$ & $7.6 \times 10^{8}$ & $1.3 \times 10^{2}$ \\
\hline $\mathcal{B}_{\text {peak }}$ peak brilliance ${ }^{\mathrm{a}}$ & $3.3 \times 10^{14}$ & $3.7 \times 10^{9}$ \\
\hline
\end{tabular}
experimental values as a reference.

a photons $/ \mathrm{s} \mathrm{mm}^{2} \mathrm{mrad}^{2} 0.1 \% \mathrm{BW}$.

characteristics have been optimized for x-ray production the wire scanner apparatus described in Sec. II D 1 is used to obtain spatial overlap of the two beams. The electron beam alignment is not adjusted after focusing so its position and shape are measured with the wire scans. The laser beam steering and wire scans are performed iteratively until the laser converges with the electron beam position. Convergence is clearly indicated due to an enhancement in secondary electron current from the wire when excited by both beams. Finally, the temporal synchronization of electron and laser pulses is achieved using the rf phase comparison method described in Sec. II D 2 and [29]. Figure 29 shows the detector signal as we changed the optical delay by scanning the trombone position (see Sec. II D 2) The bump in the signal at the beginning of the waveform corresponds to removing of the electron beamstop and allowing the electron beam to propagate to the interaction point and for the laser to turn on. The background signal of $\sim 40 \mathrm{mV}$ is due to the electron beam halo scrapping the vacuum beam pipe and creating secondary radiation (gammas and neutrons). See Sec. II E 3 for more details. 


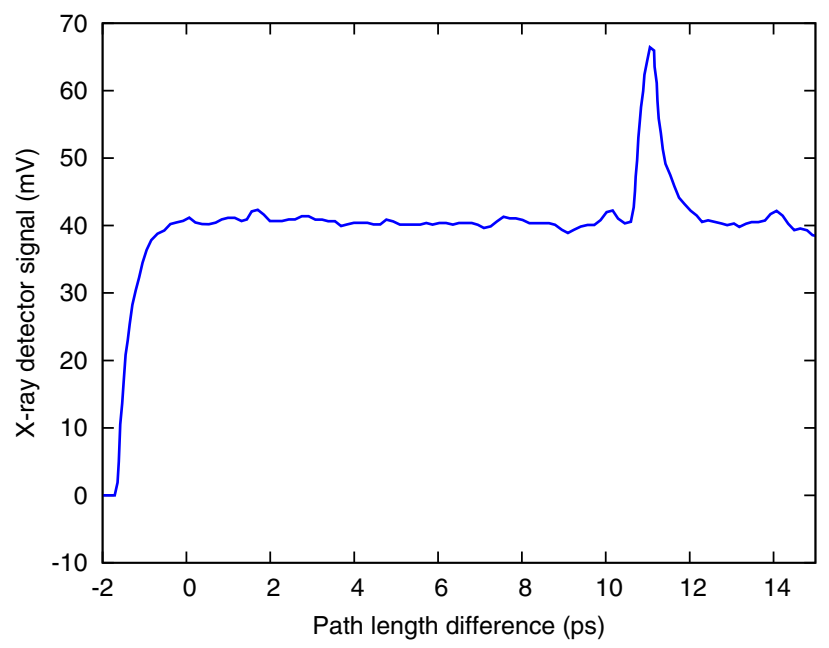

FIG. 29. X-ray signal on the PMT detector as a function of optical path length delay. When the ebeam and laser beam are temporally aligned, the x-ray signal is maximized as shown by the spike in detector voltage.

The total peak flux at the source was estimated from the PMT detector voltage, $V_{\text {PMT }}$, using Eq. (3):

$$
\mathcal{F}_{\gamma}=\frac{V_{\text {PMT }}}{T_{\text {air }} T_{H e} \Omega T_{\text {col }} Q_{Y} G_{Y} T_{Y, \text { air }} T_{\text {air,glass }} G_{\text {PMT }} R} .
$$

Here $T_{\text {air,He }}$ are the transmission coefficients through the air/helium (0.73 and 0.97), $T_{\mathrm{col}}$ is a factor accounting for the reduction in flux due to part of the active area of the YAP screen being shadowed by the lead collimator (0.52), $Q_{Y}$ is the YAP:Ce quantum efficiency (1.00), $G_{Y}$ is the gain of the YAP:Ce (250 blue photons/10 keV x-ray), $T_{\text {air,glass }}$ is the transmission of the air/glass interface, $G_{\mathrm{PMT}}$ is the gain of the PMT $\left(4.20 \times 10^{-11} \mathrm{~mA} /(\right.$ photon $/ \mathrm{sec})$ at $370 \mathrm{~nm}$ ), and $R$ is the termination resistor on the readout oscilloscope. $\Omega$ is the fraction of the solid angle subtended by the detector in the electron rest frame:

$$
\Omega=\frac{1}{2}\left[1-\cos \left(\theta_{r f}\right)\right],
$$

with $\theta_{r f}=\operatorname{atan}(\gamma * x / z)$, x being the height of the detector, and $\mathrm{z}$ the distance to the detector from the source. The average flux was then $\mathcal{F}_{\text {ave }}=\mathcal{F}_{\gamma} \tau_{m p} f_{m p}$.

\section{Comparison}

The width of the detector signal peak reflects the $\sim 2$ ps laser pulse length. Measurement of the peak x-ray signal at the detector was used to extrapolate a measured flux into all angles of $2.2 \times 10^{10}$ during the macropulse and $1.3 \times 10^{5}$ photons/s time averaged during the experiment running at $4 \mathrm{~Hz}$ with a $1.5 \mu$ s laser macropulse. The uncertainty in the measurement was $4 \%$ from instrument readout limitations.
Note that while the electron beam macropulse was $5 \mu$ s the FEL turns on slowly due to a kink in the magnetic field at the entrance of the undulator, leading to the shorter laser macropulse. The transmission of the transport/detection system (Fig. 27) and a factor accounting for the angle subtended by the detector and the angular distribution of the radiation was used to calculate the flux. To calculate this factor, the angle subtended by the detector is converted to the electron rest frame, $\theta_{\mathrm{X}}^{\prime}=\arctan \left(\gamma \tan \theta_{\mathrm{X}}\right)$, where the radiation is isotropic, and the ratio of the solid angle subtended is divided by the total solid angle to get the scaling factor $F_{\theta}=2 \pi\left(1-\cos \theta_{\mathrm{X}}^{\prime}\right) / 4 \pi$. The experimental measurements are slightly lower than the predicted values ("Experiment" column in Table II) most likely due to a slight misalignment in the electron/laser beam collision.

\section{SCIENTIFIC MILESTONES AND PLANNED UPGRADES}

A number of planned upgrades to the system are described in this section, and the expected performance enhancements are detailed in Table III.

\section{A. Microwave gun duty cycle improvements}

During operation, the cathode in the thermionic electron gun heats up due to back-bombardment from the constant flow of electrons emitted during the rf accelerating field's cycle. Limiting the length of the macropulse to about $5 \mu \mathrm{s}$ keeps the temperature in a stable range preventing damage due to overheating. The storage cavities employed in a cavity enhanced ICS sources, however, have 1/e decay

TABLE III. Current vs upgraded operating parameters for FELICIA.

\begin{tabular}{lcc}
\hline \hline Symbols and parameters & Current & Upgrade \\
\hline rf/electron pulses & & \\
$\tau_{m p}$ macropulse duration $(\mu \mathrm{s})$ & 5 & 20 \\
$f_{m p}$ macropulse frequency $(\mathrm{Hz})$ & 4 & 100 \\
$\epsilon_{n}$ normalized emittance $(\pi$-mm-mrad) & 25 & 8 \\
Laser pulses & & \\
$\mathcal{P}_{l}$ laser power (MW) & 0.3 & 2.6 \\
Interaction point & & \\
rms electron beam radius $(\mu \mathrm{m})$ & 53 & 30 \\
$\mathcal{F}$ storage cavity finesse & $\ldots$ & 2000 \\
$\mathcal{P}_{s}$ stored optical power $(\mathrm{GW})$ & $\ldots$ & 2.6 \\
Scattered x-rays $_{\mathcal{E}_{\gamma} \text { X-ray energy (keV) }}$ & \\
$\lambda_{\gamma}$ x-ray wavelength $(\AA)$ & 10 & 10 \\
$\mathcal{F}_{\gamma}$ total average X-ray flux $(\mathrm{ph} / \mathrm{s})$ & $3.0 \times 10^{5}$ & $9.2 \times 10^{11}$ \\
$\theta=1 / \gamma$ x-ray divergence angle $(\mathrm{mrad})$ & 12.6 & 12.1 \\
$\mathcal{B}_{\text {ave }}$ average brilliance & \\
$\mathcal{B}_{\text {peak }}$ peak brilliance & $1.3 \times 10^{2}$ & $3.4 \times 10^{9}$ \\
\hline \hline
\end{tabular}

${ }^{\mathrm{a}}$ photons $/ \mathrm{s} \mathrm{mm}^{2} \mathrm{mrad}^{2} 0.1 \% \mathrm{BW}$. 
times of $10 \mu \mathrm{s}$ or more. Thus if the macropulse length limitation is removed, a factor of 2 or more increase in stored cavity energy and $\mathrm{x}$-ray flux would be possible. Our technique to counteract back-bombardment (BB), already developed and tested, uses a laser prepulse incident on the cathode surface before the rf macropulse to cool the cathode surface as laser heat diffuses into the bulk [19]. The diffusive cooling counteracts the BB heating, drastically reducing the temperature rise and extending the macropulse length and ultimately increasing the $\mathrm{x}$-ray flux from these sources.

We performed numerical simulations of the cathode surface temperature including all the relevant physics: diffusion, heat deposition by bombarding electrons and laser, radiative losses, and heating by the tungsten heating element. The work function and Richardson constant of the cathode material, which vary substantially with preparation [41] and are therefore difficult to predict, were used as fit parameters, in conjunction with the measured cathode temperature. These simulations are a very accurate model of our real system and match the measured cathode current to that predicted by the thermionic emission equation. Figure 30 shows a result of these simulations. In red is the measured temperature of the cathode surface and resulting gun current pulse, limited to $5 \mu$ s by the steep temperature ramp. The blue curve shows how the diffusive cooling due to $500 \mathrm{~mJ}$ of laser heat deposited over a $40 \mu$ s laser prepulse compensates for the temperature increase due to $\mathrm{BB}$,
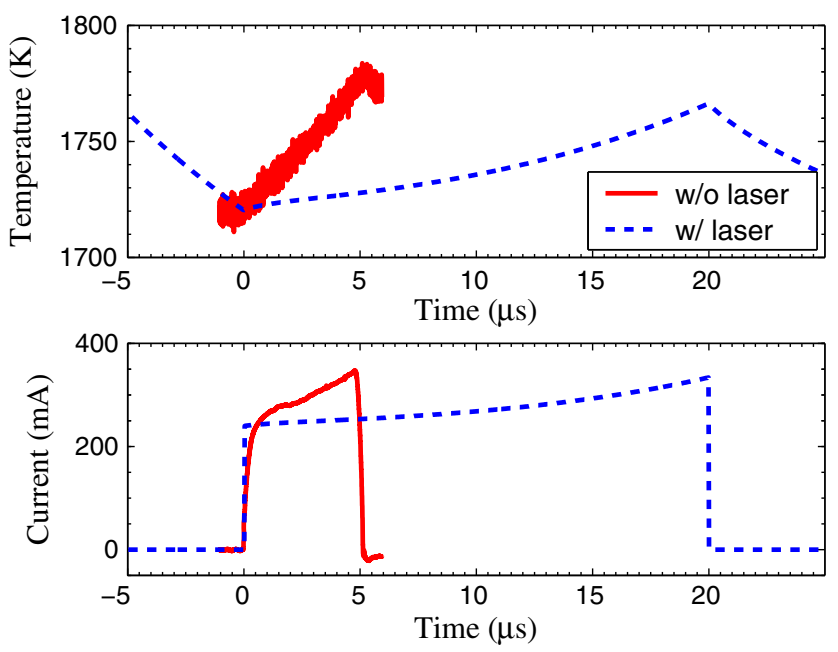

FIG. 30. Comparison of cathode surface temperature and current as a function of time with and without the laser prepulse. The red curve shows the temperature measurement of the cathode surface: back-bombardment (BB) causes a rapid increase in temperature and current, limiting the rf macropulse to $5 \mu \mathrm{s}$. The blue dotted curve shows simulation of the stabilized temperature and current from the combined cooling effect of $500 \mathrm{~mJ}$ of laser heat deposited before $\mathrm{BB}$ begins at time 0 on the plot. The rf macropulse can be extended to $20 \mu$ s with less temperature rise than the present configuration. resulting in temperature stabilization for $20 \mu \mathrm{s}$ and enabling 4 times the average output current.

A fourfold increase in average current would increase the average $x$-ray flux of the FELICIA source in two ways. First, the number of colliding electron pulses would increase, leading to a commensurate increase in number of x-ray pulses. Second, since the length of the FEL laser pulse train will increase commensurate with the electron pulse train, more pulses will be stacked in the enhancement cavity, leading to higher peak fields and more $\mathrm{x}$-ray photons per pulse.

There are two limitations to the use of this laser prepulse technique to increase the duty cycle. First, since the resonance properties of the cavity are temperature dependent, sufficient cooling will need to be devised to remove the additional heat deposited by the significantly longer rf macropulse into the normally conducting gun cavity. Second, though feasible in principle, an rf source which can deliver a $40 \mathrm{MW}, 20 \mu \mathrm{s}$ macropulse at $100 \mathrm{~Hz}$ repetition rate will need to be demonstrated.

\section{B. Optical storage cavity}

The advantages of externally pumped storage cavities for $\mathrm{x}$-ray and gamma-ray production by Compton backscattering from a tightly focused intracavity waist have been recognized for many years [42]. Storage-ring based ebeam systems, in particular, have seen significant development. However, due to their physical dimension, storage ringbased systems require optical cavities with lengths of many meters and finesses in the extreme $(30,000)$. These requirements prohibit the use of two-mirror, near-concentric cavities due to their intrinsic instability, and stable designs such as the bow-tie ring resonator have been extensively developed in their stead [43]. The availability of the $\mathrm{GHz}$ repetition rates in both the electron beam and optical source at the University of Hawaii has permitted us to reevaluate the application of two-mirror resonators (i.e., retroreflecting as opposed to ring geometries) in high luminosity $\mathrm{x}$ - and gamma-ray production.

The basic design of our system is fundamentally constrained by the macropulse structure of our electron and optical beams. In facilities that employ cw pulse trains, such as the Duke HIGS Laboratory [13], maximum circulating power is achieved by maximizing the finesse, and the $\mathrm{cw}$ pulse train can build up to this maximum steadystate power independent of the length of the cavity. In contrast, our system uses macropulses of finite duration, and the number of passes in the storage cavity is thus finite. Since the circulating power is maximized by maximizing the number of passes, our system benefits both from the $\mathrm{GHz}$ repetition rate and from shorter cavities. Our cavity length of $0.472 \mathrm{~m}$ is designed to be as short as possible while remaining above the damage threshold of the mirrors. Given a finite number of passes N, simulations show that the integrated circulating power is optimized for a cavity 


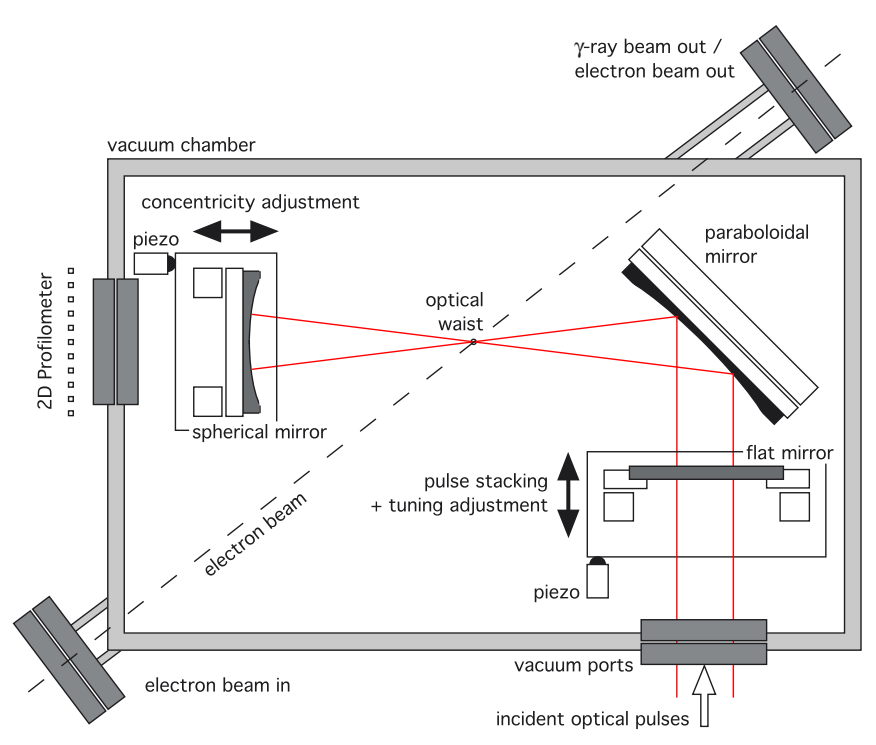

FIG. 31. Schematic design of the full-up optical storage cavity.

loss $\delta_{c}$ satisfying $\delta_{c} \mathrm{~N}=3.8$; higher values of $\delta_{c}$ (lower finesse) reduce the steady-state value of circulating power, while lower values (higher finesse) delay the steady-state regime too far beyond the duration of the macropulse. Thus, it is ultimately the finite duration of the macropulse that constrains the cavity finesse; a macropulse duration of $5 \mu$ s corresponds to $\mathrm{N}=1590$ and yields a relatively modest finesse of 2600 .

The design of our future system is based on the novel retroreflecting, three-mirror optical storage cavity described in U.S. Patent 7,532,649. The configuration of this cavity, illustrated schematically in Fig. 31, is designed to provide simultaneous, independent, and real-time control over both the cavity concentricity and pulse-stacking requirements for optimization of Compton backscattering.

As stepping stones in the development of the full-up storage cavity, we divided the effort into two simpler resonator configurations [28]. The first was a four-mirror, visible (HeNe) cavity employing two cylindrical mirrors configured to mimic the off-axis paraboloid. This resonator was used to study the microfocal and alignment properties of the resonator. The second configuration was a straight, two-mirror resonator, operable at $3 \mu \mathrm{m}$ wavelength, which was used to study the pulse stacking properties of the resonator using IR pulses from the phase-locked FEL.

Since the paraboloidal mirror is a challenging and costly optic to manufacture to the required specifications, we developed several basic capabilities by substituting a pair of low-cost cylindrical mirrors with equivalent focusing characteristics. The modified four-mirror cavity allowed us to develop the following capabilities essential to the development of the high power IR cavity: (1) the ability to achieve a diffraction limited focus using anamorphic optics by carefully balancing astigmatism and other optical aberrations; (2) the ability to precisely set the synchronous length

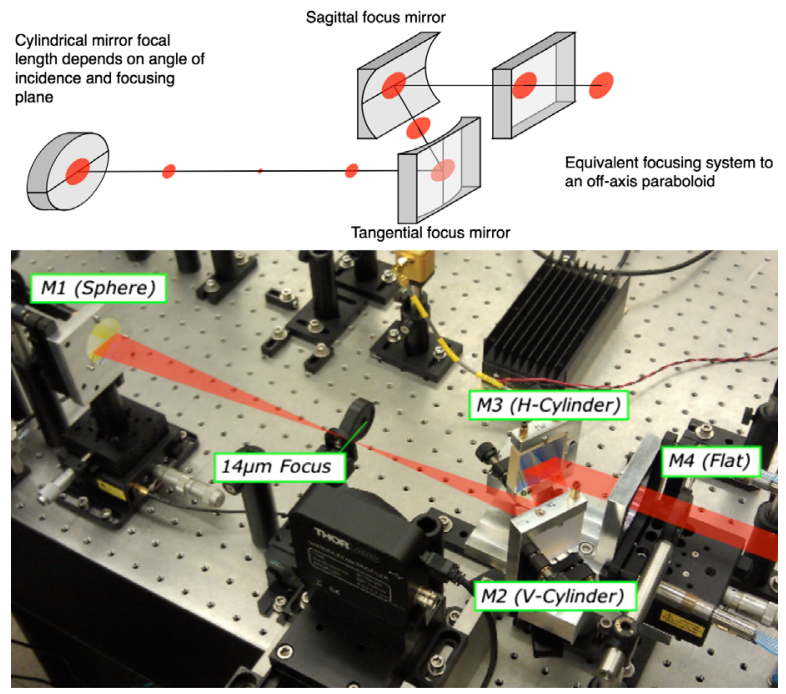

FIG. 32. Schematic design and laboratory construction of the four-mirror HeNe storage cavity.

of the cavity to match the electron pulse repetition rate; (3) the ability to lock the cavity to a single longitudinal mode using Pound-Drever-Hall stabilization; and (4) to adjust the alignment and concentricity of the cavity to obtain the required focal spot at the interaction point. The schematic configuration and laboratory construction of this cavity are illustrated in Fig. 32.

Using this resonator, we were able to demonstrate good higher order mode discrimination, together with the production of a diffraction-limited microfocus as low as $14 \mu \mathrm{m}$ radius [28], consistent with theoretical predictions for the HeNe wavelength. The observed $\mathrm{TEM}_{00}$ and higher order spatial modes are shown in Fig. 33. Since concentric optical cavities are degenerate with respect to their transverse mode structure, these results indicate that the slight deviation from concentricity required for stable operation of the storage cavity is sufficient to break the mode degeneracy, which would otherwise increase the focal spot size. The concentricity deviation of this cavity was specifically engineered to minimize the degeneracy of the first four transverse mode orders. The formation of a diffraction
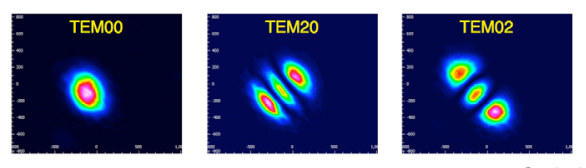

TEMO0 and Higher Order Spatial Mode Selectivity

Near diffraction limited, non-astigmatic focal spot using anamorphic focusing mirrors

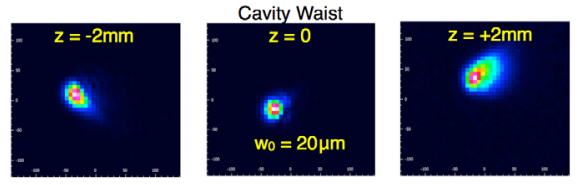

$0.12 \mathrm{~mm}$ astigmatism $\ll<\left(z_{\mathrm{R}}=2 \mathrm{~mm}\right)$

FIG. 33. Spatial mode structure in the four-mirror HeNe storage cavity. The transverse mode structure of the cavity is measured using a focal plane array sampling the cavity's out-coupled beam. 
limited microfocus at the cavity waist was verified by sampling the intracavity beam with a low-loss pellicle beam splitter and performing a z-scan measurement using a CCD beam profiler array.

One of the most important results gained from these studies was revealed by a numerical investigation into the origin of the higher-order modes oriented at $45^{\circ}$ to the axis [28], as seen in Fig. 33. This mode structure is in fact due to an astigmatic aberration which is special to the geometry of the cylindrical mirrors used in the four-mirror cavity. Once this effect was understood, we were able to reproduce its presence in ray-trace simulations of the four-mirror cavity,
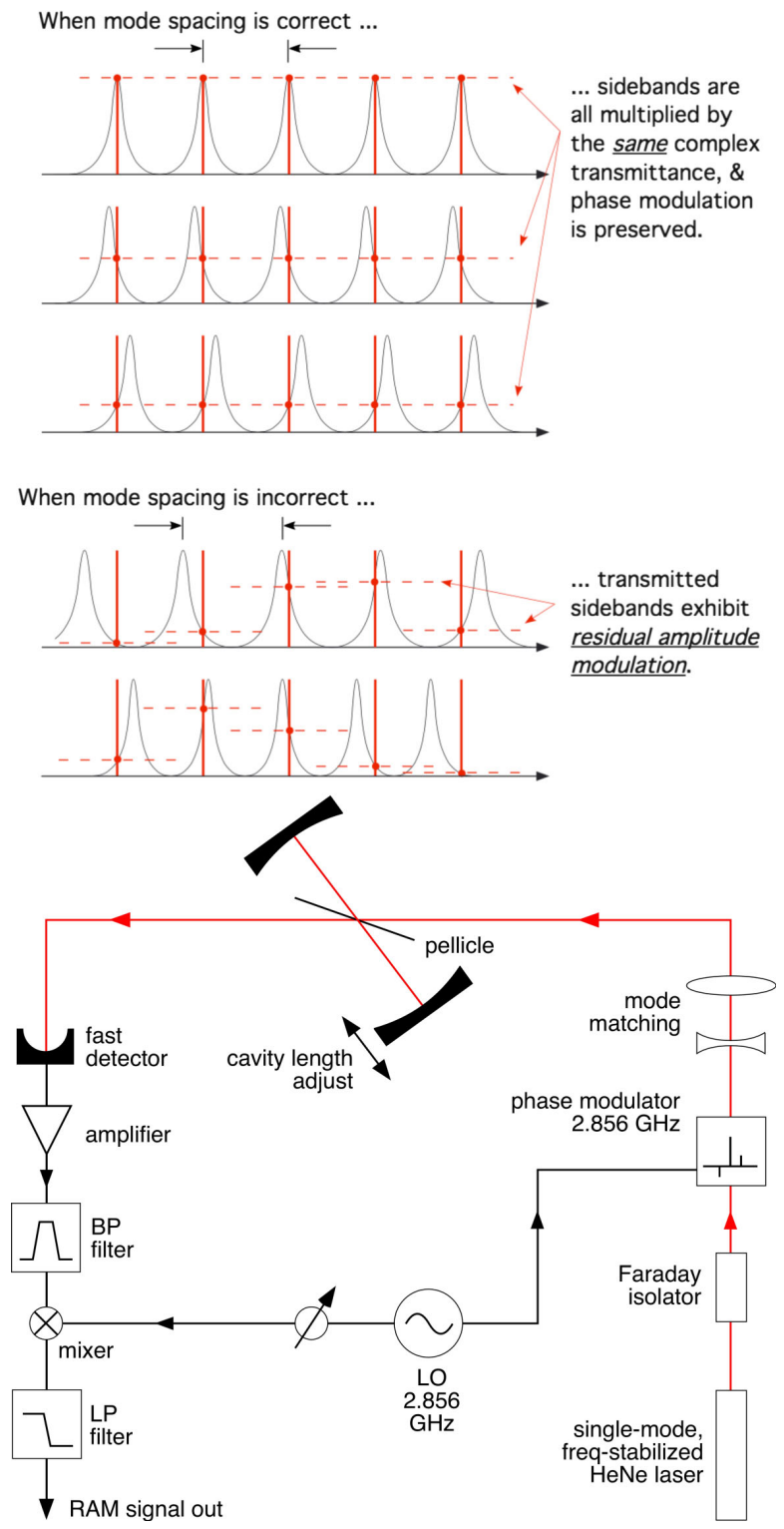

FIG. 34. Residual amplitude modulation (RAM) technique for matching the storage cavity length to the electron- and laser-pulse repetition rate: (top) concept based on elimination of residual amplitude modulation; (bottom) optoelectronic implementation using a single-mode HeNe laser. and to demonstrate that the effect is entirely absent in the three-mirror cavity using the off-axis paraboloid [28].

In parallel with the efforts to characterize the spatial (transverse mode) optical properties of the storage cavity, we used a separate two-mirror IR cavity to separately investigate the pulse-stacking (longitudinal mode) optical properties of the storage cavity pumped by a phase-locked FEL. As described in Sec. II C 1, all timing is governed by the $2.856 \mathrm{GHz}$ rf source that drives the accelerator and laser source. Thus, the storage cavity must be matched to this frequency to achieve pulse stacking. The method we developed to achieve this condition is based on phase modulation of a single mode $\mathrm{HeNe}$ beam at the rf frequency to detect residual amplitude modulation when the cavity axial mode frequency is not matched to the rf frequency [44]. The technique and its implementation are described in Fig. 34.

Using this technique, we were able to establish the correct cavity length to within $1 \mu \mathrm{m}$ in the visible storage cavity (which was highly reflecting at $\mathrm{HeNe}$ wavelength) and to within $10 \mu \mathrm{m}$ in the two-mirror infrared (IR) storage cavity (which had reduced reflectance at $\mathrm{HeNe}$ wavelength). This technique was instrumental in achieving IR pulse stacking of the FEL pulses at $3 \mu \mathrm{m}$; without it, a search for the synchronous length by scanning would have been time prohibitive given the $4 \mathrm{~Hz}$ rep rate of the FEL. The configuration of the two-mirror IR storage cavity and the observed residual amplitude modulation are shown in Fig. 35.

An important milestone in the storage cavity project was the demonstration of pulse stacking of the infrared

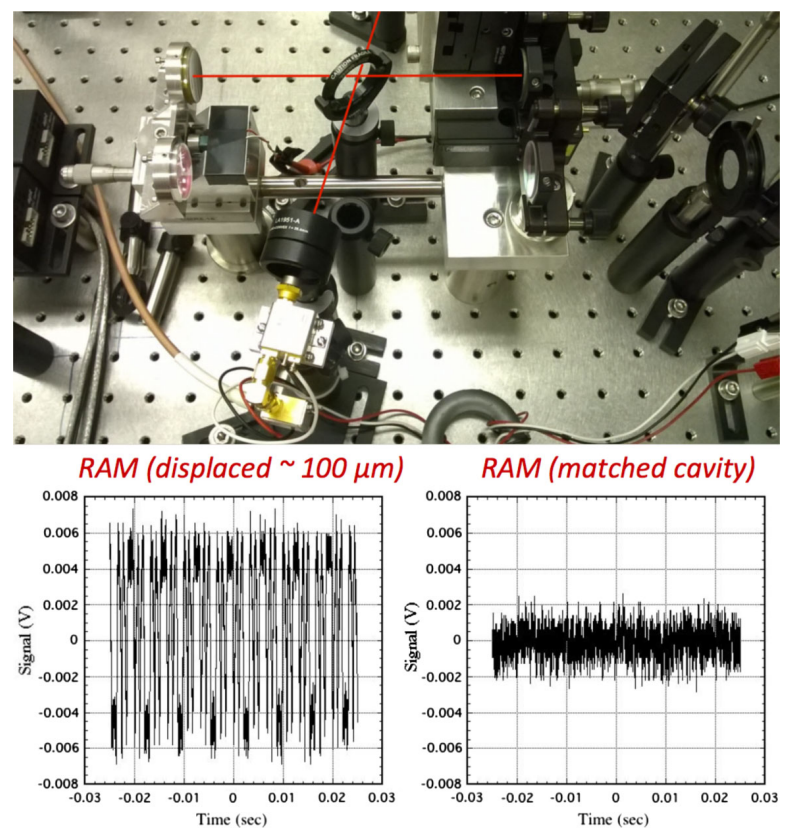

FIG. 35. Setup (top) of residual amplitude modulation (bottom) in the two-mirror IR storage cavity using a phase-modulated single-mode HeNe laser. 


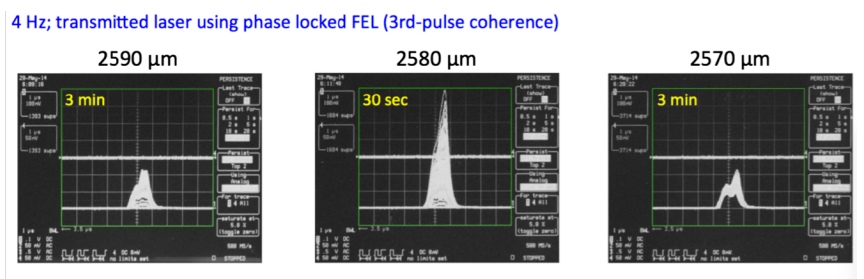

$4 \mathrm{~Hz}$; transmitted laser with no phase locking
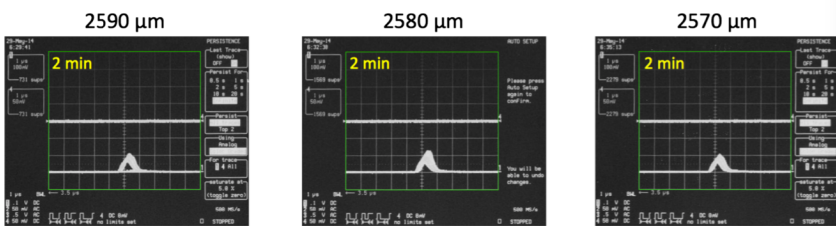

FIG. 36. Phase-locked FEL pulse stacking in the two-mirror IR storage cavity versus cavity length. The number above each panel indicates the relative cavity length variation measured with an LVDT. The time stamp at the top left corner of each panel indicates the oscilloscope persistence time used to record successive macropulses, and affects only the number of macropulses displayed in each panel.

picosecond FEL optical pulses in bench tests of the twomirror IR storage cavity, and the fundamental demonstration that pulse stacking is achievable only with phaselocked operation of the FEL. As noted previously, this mode of operation is required to establish the necessary interpulse phase coherence in the incident pump pulses. The two-mirror IR test cavity was designed to contain three circulating optical pulses, which could be coherently pumped using an FEL configuration in which every third pulse was coupled in the interferometer.

Figure 36 shows the demonstration of pulse stacking, which revealed itself in the detected signal of the transmitted pulses through the storage cavity. The first set of traces shows the cavity length discrimination of the effect when the incident pulses are phase locked. The relative macropulse heights clearly indicate the presence of pulse stacking during the macropulse. The second set of traces used FEL pulses in which phase locking was absent, and demonstrated a complete absence of pulse stacking even at the synchronous length $(2580 \mu \mathrm{m}$ linear variable differential transformer (LVDT) reading).

Future development of the storage cavity will adapt the above techniques to the IR cavity based on the three-mirror paraboloidal design, together with efforts to stabilize operation on a single longitudinal mode.

\section{Upgrading the rf system}

Although only 15 MW peak power is required by the linac to achieve the design accelerating gradient of $10-15 \mathrm{MeV} / \mathrm{m}$, additional power for driving the microwave gun and compensation for losses in the waveguide is required. In order to better match the longer pulse lengths enabled by laser cathode cooling, a pulse rf system with $40 \mathrm{MW}$ peak power over a $20 \mu$ s long pulse is desired.

Limitations on both macropulse length and repetition rate are imposed on both the electron beam, the FEL IR beam, and the resulting scattered x-ray photon output of FELICIA by the high voltage modulator and rf amplifier chain. S-band rf power at $2.856 \mathrm{GHz}$ is generated in the control room by a precision crystal oscillator whose frequency is monitored by an eight-digit counter and held to better than $10 \mathrm{kHz}$ drift from nominal accelerating frequency. The $\mathrm{cw}$ oscillator output is amplified by an intermediate klystron and then fed to a high-power klystron rated at $40 \mathrm{MW}$ peak power. A pulse-forming network stores energy supplied by the Titan-Beta high-voltage power supply discharging $20 \mathrm{kV}$ in a $7 \mu$ s-long pulse through a transformer providing the klystron beam accelerating voltage of roughly $200 \mathrm{kV}$. The rf from the intermediate $\mathrm{cw}$ klystron is gated by a fast rf PIN diode and applied to the input of the high power klystron whose output feeds rf power to both the linac and microwave gun, distributed through an evacuated waveguide network coupled by an rf ceramic window. The gun, linac, and waveguide have been processed at high power up to the limit of $4.5 \mu$ s imposed by cathode heating caused by backbombardment of electrons. The fundamental limit on pulse length output from the rf system is imposed by the pulseforming network pulse length of less than $10 \mu \mathrm{s}$. Different possibilities for future upgrades are being considered. Table III summarizes the system performance anticipated after implementation of the upgrades discussed in this paper.

\section{CONCLUSION}

The research, development, and results presented here document the progress made in the FELICIA project at the University of Hawaii. The single-pass experiment produced total average flux of $3.0 \times 10^{5}$ photons/second $\mathrm{x}$-rays at $10.9 \mathrm{keV}$ peak energy with an average brilliance of $2.0 \times 10^{7}$ photons $/ \mathrm{s} \mathrm{mm}^{2} \mathrm{mrad}^{2} 0.1 \% \mathrm{BW}$. In order to reach source brightness competitive with large synchrotron labs, we have designed and characterized an optical cavity for the $\mathrm{MkV}$ beam line. On the diagnostic front, a picosecond $\mathrm{x}$-ray detector and readout electronics were developed. These steps will lead to the realization of an x-ray source with flux capable of supporting the many different emerging applications of a compact ICS, including timeencoded differential absorption and/or differential fluorescence imaging techniques.

\section{ACKNOWLEDGMENTS}

We thank the management and support of the University of Hawaii, particularly College of Natural Sciences and Department of Physics and Astronomy, especially Professor Pui Lam, for his support. We also thank operators 
Carly Hall and Foster Ducker for their help with the daily task of conditioning. This work was funded by National Science Foundation and Department of Homeland Security Academic Research Initiative Grants No. 2010-DN-077AR1045-02 and No. 2011-DN-077-AR1055-02.

[1] W. A. Barletta and M. Borland, Report of the basic energy sciences workshop on compact light sources, Basic Energy Science Workshop Report, 2010_Rockville Hilton, Rockville, MD, USA(2010), https://science.energy.gov/ /media/ bes/pdf/reports/files/CLS.pdf.

[2] J. C. Hemminger et al., Report of the BESAC Subcommittee on Future X-ray Light Sources (2013), https:// science.energy.gov/ /media/bes/besac/pdf/Reports/Future_ Light_Sources_report_BESAC_approved_72513.pdf.

[3] N. Kikuzawa, R. Hajima, N. Nishimori, E. Minehara, T. Hayakawa, T. Shizuma, H. Toyokawa, and H. Ohgaki, Nondestructive detection of heavily shielded materials by using nuclear resonance fluorescence with a laser-compton scattering $\gamma$-ray source, Appl. Phys. Express 2, 036502 (2009).

[4] C. Settens, B. Bunday, B. Thiel, R. J. Kline, D. Sunday, C. Wang, W.-L. Wu, and R. Matyi, Critical dimension small angle X-ray scattering measurements of FinFET and 3D memory structures, Proc. SPIE Int. Soc. Opt. Eng. 8681, 86810L (2013).

[5] H. Wen, A. A. Gomella, A. Patel, S. K. Lynch, N. Y. Morgan, S. A. Anderson, E. E. Bennett, X. Xiao, C. Liu, and D.E. Wolfe, Subnanoradian X-ray phase-contrast imaging using a far-field interferometer of nanometric phase gratings, Nat. Commun. 4, 2659 (2013).

[6] A. L. Carvalho, J. Trincão, and M. J. Romão, Ligandmacromolecular interactions in drug discovery, Methods Mol. Biol. 572, 31 (2009).

[7] A. Sakdinawat and D. Attwood, Nanoscale X-ray imaging, Nat. Photonics 4, 840 (2010).

[8] J. M. J. Madey, E. B. Szarmes, M. R. Hadmack, B. T. Jacobson, J. M. D. Kowalczyk, and P. Niknejadi, Optimized cavity-enhanced x-ray sources for x-ray microscopy, Proc. SPIE Int. Soc. Opt. Eng. 8851, 88510W (2013).

[9] W. S. Graves, J. Bessuille, P. Brown, S. Carbajo, V. Dolgashev, K.-H. Hong, E. Ihloff, B. Khaykovich, H. Lin, K. Murari, E. A. Nanni, G. Resta, S. Tantawi, L. E. Zapata, F. X. Kärtner, and D. E. Moncton, Compact X-ray source based on burst-mode inverse Compton scattering at 100 kHz, Phys. Rev. ST Accel. Beams 17, 120701 (2014).

[10] A. Variola et al., in Proceedings of the 5th International Particle Accelerator Conference IPAC2014, Dresden, Germany (JACoW, Geneva, Switzerland, 2014), pp. 2062-2064, WEPRO052, DOI: 10.18429/JACoWIPAC2014-WEPRO052.

[11] E. Eggl, M. Dierolf, K. Achterhold, C. Jud, B. Günther, E. Braig, B. Gleich, and F. Pfeiffer, The munich compact light source: initial performance measures, J. Synchrotron Radiat. 23, 1137 (2016).
[12] B. Dunham et al., Record high-average current from a high-brightness photoinjector, Appl. Phys. Lett. 102, 034105 (2013).

[13] T. S. Carman, V. Litveninko, J. Madey, C. Neuman, B. Norum, P. G. O'Shea, N. R. Roberson, C. Y. Scarlett, E. Schreiber, and H.R. Weller, The TUNL-FELL inverse Compton $\gamma$-ray source as a nuclear physics facility, Nucl. Instrum. Methods Phys. Res., Sect. A 378, 1 (1996).

[14] H. A. Enge, Achromatic magnetic mirror for ion beams, Rev. Sci. Instrum. 34, 385 (1963).

[15] G. A. Westenskow and J. M. J. Madey, Microwave electron gun, Laser Part. Beams 2, 223 (1984).

[16] G. A. Westenskow, J. M. J. Madey, L. C. Vintro, and S. V. Benson, Owner's Manual for the Microwave Electron Gun, Technical Report, HEPL Technical Note TN-86-1, 1986.

[17] C. B. McKee and J. M. J. Madey, Optimization of a thermionic microwave electron gun, Nucl. Instrum. Methods Phys. Res., Sect. A 304, 386 (1991).

[18] A. H. Lumpkin, M. R. Hadmack, J. M. D. Kowalcyzk, E. B. Szarmes, and J. M. J. Madey, Initial streak camera measurements of the S-band linac beam for the University of Hawaii FEL oscillator, in Proceedings of the 35th International Free Electron Laser Conference, edited by C. Scholl and V. R. W. Schaa (JACoW, New York, NY, USA, 2013), pp. 325-328, TUPSO45, http://accelconf.web .cern.ch/AccelConf/fel2013/papers/tupso45.pdf.

[19] J. M. D. Kowalczyk and J. M. J. Madey, Back-bombardment compensation in microwave thermionic electron guns, Phys. Rev. ST Accel. Beams 17, 120402 (2014).

[20] C. Boulware, J. Corlett, K. Harkay, F. Hannon, C. Hernandez-Garcia, T. Kamps, M. Krasilnikov, B. Militsyn, T. Quast, F. Sannibale, and J. Teichert, Electron sources for future lightsources, summary and conclusions for the activities during FLS 2012, in Summary Paper from Working group Future Light Sources 2012 Workshop at ICFA Workshop on Future Light Sources, Newport News, Virginia, USA, arXiv:1307.1571.

[21] P. B. Sewell, Kimball Physics, Inc. Technical Bulletin No. LaB6-01B, 1991.

[22] H. Wiedemann, Particle Accelerator Physics, 3rd ed. (Springer, New York, 2007).

[23] B. T. Jacobson, J. M. J. Madey, and P. Niknejadi, in Proceedings of the 4th International Particle Accelerator Conference IPAC2013, Shanghai, China (JACoW, Shanghai, China, 2013), pp. 2280-2282, WEPWA072, http://accelconf.web.cern.ch/AccelConf/ipac2013/papers/ wepwa072.pdf.

[24] B. T. Jacobson, Design, Commissioning, and Operation of MkV Electron Beam Diagnostic Chicane, Ph.D. thesis, University of Hawaii at Manoa, 2011.

[25] P. Niknejadi, Electrodynamics of relativistic electron beam x-ray sources, Ph.D. thesis, University of Hawaii at Manoa, 2016.

[26] P. Niknejadi and J. M. J. Madey, Optimization of electron beam and laser pulse alignment and focusing at interaction point for a compact FEL based inverse-compton scattering $\mathrm{x}$-ray source, in Proceedings of the 7th International Particle Accelerator Conference IPAC2016 Busan, Korea 
(JACoW, Geneva, Switzerland, 2016), pp. 1881-1884, TUPOW051, DOI: 10.18429/JACoW-IPAC2016-TUPOW051.

[27] B. T. Jacobson, J. M. J. Madey, M. R. Hadmack, J. M. D. Kowalczyk, and P. Niknejadi, Modular logarithmic amplifier beam position monitor readout system at the University of Hawai'i, Proceedings of 1st International Beam Instrumentation Conference (IBIC) 2012, Tsukuba, Japan (JACoW, Geneva, Switzerland, 2012), pp. 90-94, MOPA17, http://accelconf.web.cern.ch/AccelConf/IBIC2012/papers/ mopa17.pdf.

[28] M. R. Hadmack, An optical storage cavity-based, Compton-backscatter x-ray source using the MKV free electron laser, Ph.D. thesis, University of Hawaii at Manoa, 2012.

[29] M. R. Hadmack, B. T. Jacobson, J. M. D. Kowalczyk, B. R. Lienert, J. M. J. Madey, and E. B. Szarmes, Electron bunch energy and phase feed-forward stabilization system for the Mark V RF-linac free-electron laser, Rev. Sci. Instrum. 84, 063302 (2013).

[30] Z. Li, T. O. Raubenheimer, and R. Miller, Beam loading compensation for the NLC low frequency linacs, in Proceedings of the 18th Particle Accelerator Conference, New York, 1999, edited by A. Luccio and W. W. MacKay (IEEE, New York, 1999), Vol. 5, pp. 3483-3485, http:// accelconf.web.cern.ch/AccelConf/p99/PAPERS/FRA43 .PDF.

[31] M. R. Hadmack, E. B. Szarmes, J. M. J. Madey, and J. M. D. Kowalczyk, Temporal synchronization of $\mathrm{GHz}$ repetition rate electron and laser pulses for the optimization of a compact inverse-Compton scattering x-ray source, Nucl. Instrum. Methods Phys. Res., Sect. A 772, 1 (2015).

[32] G. R. Edlin, W. R. Jones, and J. F. Perkins, Phase locked rf linac free electron laser, U.S. Patent No. 4,748,629 (1988).

[33] E. B. Szarmes and J. M. J. Madey, Free-electron laser oscillator for simultaneous narrow spectral resolution and fast time resolution spectroscopy, U.S. Patent No. 5,130,994 (1992).

[34] D. Oepts, R. J. Bakker, D. A. Jaroszynski, A. F. G. van der Meer, and P. W. van Amersfoort, Induced and Spontaneous Interpulse Phase Locking in a Free-Electron Laser, Phys. Rev. Lett. 68, 3543 (1992).

[35] E. B. Szarmes, S. V. Benson, and J. M. J. Madey, Mode control on short-pulse FELs using a Michelson-mirror resonator, Nucl. Instrum. Methods Phys. Res., Sect. A 296, 98 (1990).
[36] E. B. Szarmes, A. D. Madden, and J. M. J. Madey, Optical phase locking of a $2.86-\mathrm{GHz}$ harmonically modelocked free-electron laser, J. Opt. Soc. Am. B 13, 1588 (1996).

[37] M. R. Hadmack and E. B. Szarmes, Scanning wire beam position monitor for alignment of a high brightness inverse-compton x-ray source, in Proceedings of 2 nd International Beam Instrumentation Conference (IBIC) 2013, Oxford, UK (JACoW, Geneva, Switzerland, 2013), pp. 856-859, WEPF21, http://accelconf.web.cern.ch/ AccelConf/IBIC2013/papers/wepf21.pdf.

[38] R. I. Cutler, J. Owen, and J. Whittaker, Performance of wire scanner beam profile monitors to determine the emittance and position of high power $\mathrm{CW}$ electron beams of the NBS los alamos racetrack microtron, in Proceedings of the 1987 Particle Accelerator Conference, edited by E. R. Lindstrom and L. S. Taylor (IEEE, Washington, DC, 1987), Vol. 625, http://accelconf.web.cern.ch/AccelConf/ p87/PDF/PAC1987_0625.pdf.

[39] M. C. Ross, J. T. Seeman, E. Bong, L. Hendrickson, D. McCormick, and L. Sanchez-Chopitea, Wire scanners for beam size and emittance measurements at the SLC, in Proceedings of the 1991 Particle Accelerator Conference, San Francisco, CA, 1991, edited by L. Lizama and J. Chew (IEEE, New York, 1991), pp. 1201-1203, http://accelconf .web.cern.ch/AccelConf/p91/PDF/PAC1991_1201.PDF.

[40] G. A. Krafft and G. Priebe, Compton sources of electromagnetic radiation, Rev. Accel. Sci. Techol. 03, 147 (2010).

[41] H. Ahmed and A. N. Broers, Lanthanum hexaboride electron emitter, J. Appl. Phys. 43, 2185 (1972).

[42] G. A. Krafft and G. Priebe, Early milestones in the evolution of accelerators, Rev. Accel. Sci. Techol. 01, 1 (2008).

[43] H. Shimizu, A. Aryshev, Y. Higashi, Y. Honda, and J. Urakawa, Development of a 4-mirror optical cavity for an inverse Compton scattering experiment in the STF, Nucl. Instrum. Methods Phys. Res., Sect. A 745, 63 (2014).

[44] A. Araya, S. Telada, K. Tochikubo, S. Taniguchi, R. Takahashi, K. Kawabe, D. Tatsumi, T. Yamazaki, S. Kawamura, S. Miyoki, S. Moriwaki, M. Musha, S. Nagano, M.-K. Fujimoto, K. Horikoshi, N. Mio, Y. Naito, A. Takamori, and K. Yamamoto, Absolute-length determination of a long-baseline Fabry-Perot cavity by means of resonating modulation sidebands, Appl. Opt. 38, 2848 (1999). 